\title{
DIFFERENTIAL GAMES FOR STOCHASTIC PARTIAL DIFFERENTIAL EQUATIONS
}

\author{
W.H. FLEMING AND M. NISIO
}

\section{Introduction}

In this paper we are concerned with zero-sum two-player finite horizon games for stochastic partial differential equations (SPDE in short). The main aim is to formulate the principle of dynamic programming for the upper (or lower) value function and investigate the relationship between upper (or lower) value function and viscocity solution of min-max (or max-min) equation on Hilbert space.

Let us consider SPDE (1.1), a so-called controlled Zakai-equation, arising from zero-sum two-player stochastic differential game with partial information,

$$
\begin{aligned}
d \xi(x, t)= & {\left[\sum _ { i j = 1 } ^ { n } \frac { \partial } { \partial x _ { i } } \left(\sigma^{i j}(x, Y(t), Z(t)) \frac{\partial}{\partial x_{i}} \xi(x, t)\right.\right.} \\
& \left.+\sum_{i=1}^{n} \gamma^{i}(x, Y(t), Z(t)) \frac{\partial}{\partial x_{i}} \xi(x, t)-c \xi(x, t)\right] d t \\
& +\sum_{j=1}^{m} f^{\jmath}(x, Y(t), Z(t)) \xi(x, t) d W_{\jmath}(t)
\end{aligned}
$$

with initial condition

$$
\xi(x, 0)=\eta(x) .
$$

The pay-off function $J$ is defined by

$$
J(t, \eta, Y, Z, g)=E \int_{0}^{t} h(\xi(s), Y(s), Z(s)) d s+g(\xi(t)), \quad t \leq T
$$

where $T$ is a given positive number and $h(\cdot, y, z)$ and $g$ are functions on Hilbert space. Moreover, $W=\left(W_{1}, \cdots, W_{m}\right)$ is a standard $m$-dimensional Brownian motion and $Y$ and $Z$ are admissible controls for player I and II respectively, (Definition 2.1).

Received February 24, 1992 
According to W.H. Fleming and P.E. Souganidis [4], we introduce a strategy (Definition 4.2) and formulate a notion of upper (or lower) value function (see (6.2)). When $h$ and $g$ are tame functions, we can prove the principle of dynamic programming (see (5.40) and Theorem 6.2)). This fact implies that the upper (or lower) value function turns out to be a viscosity solution of the min-max (or $\max -\min )$ equation corresponding to (1.1) and (1.2).

Here we modify Lions' definition of viscosity solution [8, Part 2], which seems suitable for our problems. If a viscosity solution is unique, then the Isaacs' condition yields the existence of value of game. The finite dimensional case, namely differential game for stochastic differential equations, is investigated in [4] and W.H. Fleming and P.E. Souganidis proved that the upper value function equals the Elliott-Kalton value and turns out to be the unique viscosity solution of the min-max equation. But, in our case, the uniqueness problem is still open and we will consider a simple example and remark on its uniqueness problem in Section 8.

Recently H. Ishii [6] introduced a slightly different notion of viscosity solution and proved the unique existence of solution for nonlinear second order partial differential equations, including the min-max one. But it seems to be still open, whether the upper value function satisfies the min-max equation in Ishii's sense.

In Section 2, we recall some results on SPDE for later use. In particular, the continuity with respect to time will be studied in Section 3 . Since the lower value function is investigated in the same way as the upper one, we will only consider the upper one. Applying semi-discretization arguments, we introduce $\pi$-admissible strategy (see Definition 4.2) and the upper value function $V_{\pi}$. If the terminal function is tame, then $\left\{V_{\pi}, \pi=\right.$ partition of $\left.[0, T]\right\}$ is compact (Theorem 4.1) and the principle of dynamic programming will be proved in Section 6 . We will show that the upper value function turns out to be a viscosity solution of the min-max equation in Section 7.

\section{Preliminaries}

Let $\mathscr{Y}$ and $\mathscr{Z}$ be convex compact subsets of $R^{p}$ and $R^{q}$ respectively. $W$ denotes a standard $m$-dimensional Brownian motion, defined on a probability space $(\Omega, \mathscr{F}, P)$. For simplicity, we assume $m=1$. Put $\mathscr{F}_{t}=\sigma$-field generated by $\{W(s), s \leq t\}$.

First we will define control and strategy, according to [4]. 
Derinitiol 2.1. An admissible control process $Y$ (resp. $Z$ ) for player I (resp. II) is an $\mathscr{F}_{t}$-progressively measurable process taking values $\mathscr{Y}$ (resp. $\mathscr{Z}$ ). The set of all admissible controls for player I (resp. II) is denoted by $\mathcal{M}($ resp. $\mathcal{N}$ ).

We say that $Y$ and $Y^{*}(\in \mathcal{M})$ are the same on $[t, s]$ and we write $Y=Y^{*}$ on $[t, s]$, if

$$
P\left(Y=Y^{*} \text { a.e. on }[\mathrm{t}, \mathrm{s}]\right)=1 \text {. }
$$

A similar convention is assumed to hold for elements of $\mathcal{N}$.

Definition 2.2. An admissible strategy $\alpha$ (resp. $\beta$ ) for player I (resp. II) is a mapping $\alpha ; \mathcal{N} \rightarrow \mathcal{M}$ (resp. $\beta ; \mathcal{M} \rightarrow \mathcal{N}$ ), such that if $Z=Z^{*}$ (resp. $Y=Y^{*}$ ) on $[0, s]$ then $\alpha(Z)=\alpha\left(Z^{*}\right)$ (resp. $\beta(Y)=\beta\left(Y^{*}\right)$ ) on $[0, s]$ for any $s \in[0, T]$. The set of all admissible strategies of player I (resp. II) is denoted by $\Gamma$ (resp. $\Delta$ ).

Let $H^{p}$ be a Sobolev space of functions whose generalized derivatives up to the order $p$ belong to $L^{2}\left(R^{n}\right)$. Denote its inner product and norm by $(,)_{p}$ and \|\|$_{p}$ respectively, for $p=0, H^{0}=H,(,)_{0}=($,$) and \|\|_{0}=\|\|$ for simplic. ity.

For $Y \in \mathcal{M}$ and $Z \in \mathcal{N}$, we consider $\operatorname{SPDE}$ (1.1) with $m=1$, namely

$$
\left\{\begin{aligned}
d \xi(x, t)= & \left(\partial_{i}\left(\sigma^{i j}(x, Y(t), Z(t)) \partial_{\imath} \xi(x, t)\right)\right. \\
& \left.+\gamma^{i}(x, Y(t), Z(t)) \partial_{i} \xi(x, t)-c \xi(x, t)\right) d t \\
& +f(x, Y(t), Z(t)) \xi(x, t) d W(t), \quad x \in R^{n}, t>0, \\
& \xi(0)=\eta \in L^{2}\left(R^{n}\right),
\end{aligned}\right.
$$

where $\partial_{\imath}=\frac{\partial}{\partial x_{\imath}}$ and we employ the convention of summation over repeated indices.

Definition 2.3. We say that $\mathscr{F}_{t}$-progressively measurable process $\xi$ is a solution of (2.1), if

$$
\text { (i ) } \xi \in L^{2}\left(\Omega \times(0, T] ; H^{1}\right) \cap L^{2}(\Omega ; C([0, T] ; H))
$$

and

(ii) for $t \in[0, T]$ and $\zeta \in C_{0}^{\infty}\left(R^{n}\right)(=$ infinitely differentiable function with compact support),

$$
(\xi(t), \zeta)=(\eta, \zeta)-\int_{0}^{t}\left(\sigma^{i \jmath}(Y(s), Z(s)) \partial_{\jmath} \xi(s), \partial_{\imath} \zeta\right) d s
$$




$$
\begin{aligned}
& +\int_{0}^{t}\left(\gamma^{i}(Y(s), Z(s)) \partial_{j} \xi(s)-c \xi(s), \zeta\right) d s \\
& +\int_{0}^{t}(f(Y(s), Z(s)) \xi(s), \zeta) d W(s), \text { with probability } 1
\end{aligned}
$$

Namely we study the Zakai equation in the frame work of [7] and [10]. Since this definition is consistent with a weak solution [1] of partial differential equation, we can split (2.1) into two parts to obtain suitable evaluations (see (3.9)).

Hereafter we always assume the conditions (A1) (A4) below.

(A1) $\sigma^{i j}(\quad, y, z), \gamma^{i}(\quad, y, z), f(\quad, y, z) \in C^{4}\left(R^{n}\right)$ and

$$
\sup _{(y, z) \in \mathscr{y} \times \mathscr{Z}}\|g(., y, z)\|_{C^{4}\left(R^{n}\right)} \leq b<\infty \text {, for } g=\sigma^{i j}, \gamma^{i}, f \text {. }
$$

(A2) Lipshitz condition; there is a positive number $l=l_{g}$ such that

$$
\begin{aligned}
& \left|g(x, y, z)-g\left(x^{\prime}, y^{\prime}, z^{\prime}\right)\right| \\
& \leq l\left(\left|x-x^{\prime}\right|+\left|y-y^{\prime}\right|+\left|z-z^{\prime}\right|\right) \text { for } g=\sigma^{i,}, \gamma^{\prime}, f
\end{aligned}
$$

where $\mid$ = Euclidian norm.

(A3) uniformly elliptic; $\sigma^{i j}=\sigma^{j i}$ and there is a positive number $\tilde{\lambda}_{0}$ such that, for any $(x, y, z) \in R^{n} \times \mathscr{Y} \times \mathscr{Z}$

$$
\sum_{i j=1}^{n} \sigma^{i j}(x, y, z) \theta_{i} \theta_{j} \geq \tilde{\lambda}_{0}|\theta|^{2}, \quad \text { for } \theta=\left(\theta_{1}, \ldots, \theta_{n}\right) \in R^{n}
$$

(A4) $c$ is a non-negative constant.

First we recall Theorem 2.1, due to N.V. Krylov and B.L. Rozovskii [5] and E. Pardoux [8], in our convenient form.

THEOREM 2.1. There exists a unique solution $\xi=\xi(, \eta, Y, Z)$ of (2.1) and the following properties hold

$$
\begin{gathered}
E\left(\sup _{t \leq T}\|\xi(t, \eta, Y, Z)\|^{2}\right) \leq K\|\eta\|^{2}, \\
E\left(\int_{0}^{T}\|\xi(t, \eta, Y, Z)\|_{1}^{2} d t\right) \leq K\|\eta\|^{2} \\
E\left(\|\xi(t, \eta, Y, Z)\|^{2 l} \leq K\|\eta\|^{2 l}, \quad \text { for } t \in[0, T], l=2,3,4,\right.
\end{gathered}
$$

with a constant $K$ depending only on $T, \tilde{\lambda}_{0}$ and the bound of coefficients. 
Since the linearlity of equation (2.1) yields, with probalility 1 ,

$$
\xi(t, \eta, Y, Z)-\xi(t, \tilde{\eta}, Y, Z)=\xi(t, \eta-\tilde{\eta}, Y, Z) \text { for } t \in[0, T]
$$

the solution depends on the initial data continuously.

Let us put

$$
\begin{aligned}
& L(y, z) \zeta=\partial_{i}\left(\sigma^{\prime \prime}(, y, z) \partial_{,} \zeta\right)+\gamma^{\imath}(, y, z) \partial_{i} \zeta, \\
& L(t, Y, Z)=L(Y(t), Z(t)), L(t, Y, \beta)=L(t, Y, \beta(Y)), \\
& L(t, \alpha, Z)=L(t, \alpha(Z), Z), f(t, Y, Z)=f(\quad, Y(t), Z(t)) .
\end{aligned}
$$

Then (A3) implies the coercive condition, namely there is $\lambda_{0}>0$, such that

$$
-\langle\zeta, L(y, z) \zeta\rangle+\lambda_{0}\|\zeta\|^{2} \geq 0, \text { for } \zeta \in H^{1} \text { and }(y, z) \in \mathscr{Y} \times \mathscr{Z},
$$

where $\langle\rangle=$, duality pairing between $H^{1}$ and $H^{-1}$ under $H=H^{*}$ (= dual space of $H$ ).

Next we consider SPDE (2.8) below

$$
\left\{\begin{aligned}
d \zeta(t) & =(L(t, Y, Z) \zeta(t)-c \zeta(t)) d t+f(t, Y, Z) \zeta(t) d W(t)+F(t) d t \\
& +G(t) d W(t), \text { for } t>0, \\
\zeta(0) & =0,
\end{aligned}\right.
$$

where $F$ and $G$ are $\mathscr{F}_{t}$-progressively measurable and satisfy

$$
E\left(\int_{0}^{T}\|F(t)\|^{2} d t\right)<\infty \text { and } E\left(\int_{0}^{T}\|G(t)\|_{1}^{2} d t\right)<\infty
$$

Then, there is a unique solution $\zeta \in L^{2}\left(\Omega \times[0, T] ; H^{1}\right) \cap L^{2}(\Omega ; C([0, T] ; H))$ and the following evaluations hold [7],

$$
\begin{gathered}
E\left(\sup _{t \leq T}\|\zeta(t ; Y, Z)\|^{2}\right) \leq \tilde{K} \int_{0}^{T} E\left(\|F(t)\|^{2}+\|G(t)\|_{1}^{2}\right) d t \\
E\left(\int_{0}^{T}\|\zeta(t ; Y, Z)\|_{1}^{2} d t\right) \leq \tilde{K} \int_{0}^{T} E\left(\|F(t)\|^{2}+\|G(t)\|_{1}^{2}\right) d t
\end{gathered}
$$

with a constant $\tilde{K}$ depending only on $T, \tilde{\lambda}_{0}$ and the bound of coefficients.

Now we will recall the evaluation of negative norm \|\|$_{-2}\left(=\|\|_{H^{-2}}\right)$ due to P.L. Lions [8]. Let $\zeta \in C^{\infty}\left(R^{n}\right)$ satisfy the following condition;

$\zeta=0$ on $\left(|x| \leq \frac{1}{2}\right), \zeta=1$ on $(|x| \geq 1)$ and $0 \leq \zeta(x) \leq 1$ on $R^{n}$ 
Put $\zeta_{R}(x)=\zeta\left(\frac{x}{R}\right)$. Then we have

THEOREM 2.2 [8]. There exists a constant $K^{*}$, which is independent of $Y$ and $Z$, such that

$E\left\|\xi\left(t,\left(1-\zeta_{R}\right) \eta, Y, Z\right)\right\|_{-2}^{2} \leq K^{*}\left\|\left(1-\zeta_{R}\right) \eta\right\|_{-2}^{2}, \quad$ for $t \in[0, T], R>0$.

Moreover, for any $e \in H^{2}$,

$$
E|(\xi(t, \eta, Y, Z), e)| \leq K^{*}\left\|\left(1-\zeta_{R}\right) \eta\right\|_{-2}\|e\|_{2}+\left(\varepsilon_{R}(e)+\frac{K}{R}\right)\|\eta\|
$$

where $\varepsilon_{R}(e)=\int_{|x|>(R / 2)} e(x)^{2} d \dot{x}$.

Later, we will see that (2.12) implies weakly sequential continuity of value function.

Remark. Let $\xi_{0}$ be a solution of (2.1) with $c=0$. Then we can easily see

$$
\xi(t, \eta, Y, Z)=e^{-c t} \xi_{0}(t, \eta, Y, Z) .
$$

So, we employ $\xi_{0}$ instead of $\xi$, when we stress $c=0$.

\section{Continuity with respect to time}

In this section we study continuous dependency of a solution on time. Fixing $Y$ and $Z$, we set $L(t, \omega)=L(t, Y(\omega), Z(\omega))$. Suppose $v ; R^{n} \times \Omega \rightarrow R^{1}$ satisfies the condition,

$$
v(x, \omega) \exp \left(-r|x|^{2}\right) \in H \quad \text { for all } \omega \in \Omega,
$$

with a constant $r \geq 0$.

We consider the Cauchy problem

$$
\left\{\begin{array}{l}
\frac{\partial u}{\partial t}=L(t, \omega) u \text { for } t>s, \\
u(s)=v(\omega) .
\end{array}\right.
$$

Since coefficients $\sigma^{i j}, \gamma^{i}$ and $f$ of $L(t, \omega)$ may not be continuous in $t$, we employ a weak solution [1] of (3.2) and recall the following theorem, due to D.G. Aronson [1]. 
THEOREM 3.1. There exists a unique weak fundamental solutlon for $L(\omega)$, say $\Gamma(x, t, y, s ; \omega)$, and $(3.3) \sim(3.6)$ hold.

$$
u(x, t ; \omega)=\int_{R_{n}} \Gamma(x, t, y, s ; \omega) v(y, \omega) d y
$$

is a unique solution of (3.2)

$$
\int_{R_{n}} \Gamma(x, t, y, s ; \omega) d y=1
$$

$$
u(x, t ; \omega)=\int_{R^{n}} \Gamma(x, t, y, \theta ; \omega) u(y, \theta ; \omega) d y, \quad \text { for } \theta \in[s, t] .
$$

(3.6) There exist positive numbers $a_{1}, a_{2}$ and $p$, independent of $\omega$, such that

$$
p^{-1} g_{1}(x-y, t-s) \leq \Gamma(x, t, y, s ; \omega) \leq p g_{2}(x-y, t-s)
$$

where

$$
g_{i}(x, \theta)={\sqrt{\left(4 \pi \theta a_{i}\right)^{-n}}}^{-n} \exp \left(-|x|^{2} /\left(4 \theta a_{\imath}\right)\right), \quad i=1,2
$$

It follows from (3.4) and (3.5) that

$$
\begin{aligned}
\| u(t, \omega)- & u(\theta, \omega) \|^{2}=\int\left[\int \Gamma(x, t, y, \theta ; \omega)(u(y, \theta, \omega)-u(x, \theta, \omega)) d y\right]^{2} d x \\
& \leq p \iint g_{2}(x, t-\theta)(u(y+x, \theta, \omega)-u(y, \theta, \omega))^{2} d y d x \\
& =p \int g_{2}(x, t-\theta)\|u(\cdot+x, \theta, \omega)-u(\cdot, \theta, \omega)\|^{2} d x
\end{aligned}
$$

and, denoting $\Delta_{x} u(\cdot)=u(\cdot+x)-u(\cdot)$,

$$
\|u(t, \omega)-u(\theta, \omega)\|^{2} \leq p \int g_{2}(x, t-\theta)\left\|\Delta_{x} u(\theta, \omega)\right\|^{2} d x
$$

Let us set $\xi_{0}(t, \eta, \omega)=\xi_{0}(t, \eta, Y, Z, \omega)$ and $G(t, \omega)=f(Y(t, \omega)$, $Z(t, \omega)) \xi_{0}(t, \eta, \omega)$, for simplicity, and define $X_{1}$ and $X_{2} \in L^{2}(\Omega ; C([s, T] ; H))$ as follows,

$$
\left\{\begin{array}{l}
d X_{1}(t, \omega)=L(t, \omega) X_{1}(t, \omega) d t, \text { for } t>s \\
X_{1}(s, \omega)=\xi_{0}(s, \eta, \omega)
\end{array}\right.
$$

and 


$$
\left\{\begin{array}{l}
d X_{2}(t)=L(t, Y, Z) X_{2}(t) d t+G(t) d W(t), \text { for } t>s \\
X_{2}(s)=0 .
\end{array}\right.
$$

Since $\xi_{0}(s, \eta, \omega) \in H,(3.3)$ asserts

$$
\left.X_{1}(x, t, \omega)=\int_{R^{n}} \Gamma(x, t, y, s ; \omega) \xi_{0}(y, s, \eta ; \omega)\right) d y .
$$

Hence (3.7) implies

$$
\left\|X_{1}(t, \omega)-\xi_{0}(s, \eta, \omega)\right\|^{2} \leq p \int g_{2}(x, t-s)\left\|\Delta_{X} \xi_{0}(s, \eta, \omega)\right\|^{2} d x .
$$

On the other hand, (2.7) yields

$$
E\left\|X_{2}(t)\right\|^{2} \leq \lambda_{0} \int_{s}^{t} E\left\|X_{2}(\theta)\right\|^{2} d \theta+\int_{s}^{t} E\|G(\theta)\|^{2} d \theta .
$$

Thus, using (2.3), we have

$$
\begin{aligned}
E\left\|X_{2}(t)\right\|^{2} \leq \int_{s}^{t} & \exp \left(\lambda_{0}(t-\theta)\right) E\|G(\theta)\|^{2} d \theta \\
& \leq K|f|_{\infty}^{2}\|\eta\|^{2}\left[\exp \left(\lambda_{0}(t-s)\right)-1\right] \lambda_{0}^{-1},
\end{aligned}
$$

and $X(t)=X_{1}(t)+X_{2}(t)$ satisfies SPDE (3.12) below,

$$
\left\{\begin{array}{l}
d X(t)=L(t, Y, Z) X(t) d t+f(t, Y, Z) X(t) d W(t) \\
X(s)=\xi_{0}(s, \eta, Y, Z)
\end{array}\right.
$$

Hence, by the uniqueness of solution, $X$ coincides with $\xi_{0}$,

$$
X(t)=\xi_{0}(t, \eta, Y, Z), \quad t \geq s, \quad \text { with probablility } 1 .
$$

Noting $\xi_{0}(t, \eta, Y, Z)-\xi_{0}(s, \eta, Y, Z)=X_{1}(t)-X_{1}(s)+X_{2}(t)$, we get

$$
E\left\|\xi_{0}(t, \eta, Y, Z)-\xi_{0}(s, \eta, Y, Z)\right\|^{2}
$$

$\leq 2 p \int g_{2}(x, t-s) E\left\|\Delta_{X} \xi_{0}(s, \eta, Y, Z)\right\|^{2} d x+2 K|f|_{\infty}^{2}\left[\exp \left(\lambda_{0}(t-s)\right] \lambda_{0}^{-1}\|\eta\|^{2}\right.$.

Hereafter $k_{i}$ stands for a constant independent of $Y$ and $Z$. Let us evaluate $\Delta_{x} \xi_{0}$. Putting $q_{x}(t)=\Delta_{x} \xi_{0}(t, \eta, Y, Z)$, we can derive the equation (3.15) below, 


$$
\left\{\begin{aligned}
d q_{x}(t)= & L(t, Y, Z) q_{x}(t) d t+f(t, Y, Z) q_{x}(t) d W(t) \\
& +\left[\partial_{i}\left(\Delta_{x} \sigma^{i j}(t, Y, Z)\right) \partial_{j} \tilde{X}(t)+\Delta_{x} \gamma^{i}(t, Y, Z) \partial_{i} \tilde{X}(t)\right] d t \\
& +\Delta_{x} f(t, Y, Z) \tilde{X}(t) d W(t) \\
q_{x}(t)= & \Delta_{x} \eta
\end{aligned}\right.
$$

where $\tilde{X}(t)=\xi_{0}(\cdot+x, t, \eta, Y, Z)$. Applying the standard arguments, we get

$$
E\left\|q_{x}(t)\right\|^{2} \leq k_{1}\left(\left\|\Delta_{x} \eta\right\|^{2}+|x|^{2}\|\eta\|^{2}\right) \quad \text { for } x \in R^{n} .
$$

Combining (3.16) with (3.14), we have

$$
\begin{gathered}
E\left\|\xi_{0}(t, \eta, Y, Z)-\xi_{0}(s, \eta, Y, Z)\right\|^{2} \\
\leq k_{2}\left[\int g_{2}(x, t-s)\left\|\Delta_{x} \eta\right\|^{2} d x+(t-s)\|\eta\|^{2}\right] .
\end{gathered}
$$

Since $\left\|\Delta_{x} \eta\right\|$ tends to 0 , as $x \rightarrow 0$, the modulus of continuity in $t$ is independent of $Y$ and $Z$, but depends on the initial data $\eta$. Applying the same arguments as $[8$, part 2], we obtain

$$
E\left\|\xi_{0}(t, \eta, Y, Z)-\xi_{0}(s, \eta, Y, Z)\right\|_{-1}^{2} \leq k_{3}|t-s|\|\eta\|^{2} .
$$

The above evaluation together with (2.13) yields the following proposition,

Proposition 3.1. There is a constant $C_{1}$ such that, for $Y \in M, Z \in \mathcal{N}$ and $\eta \in H$

$E\|\xi(t, \eta, Y, Z)-\xi(s, \eta, Y, Z)\|^{2} \leq C_{1}\left(|t-s|\|\eta\|^{2}+\int g_{2}(x, t-s)\left\|\Delta_{X} \eta\right\|^{2} d x\right)$

$$
\begin{gathered}
E\|\xi(t, \eta, Y, Z)-\xi(s, \eta, Y, Z)\|_{-1}^{2} \leq C_{1}|t-s|\|\eta\|^{2} \\
E\|\xi(t, \eta, Y, Z)-\xi(s, \eta, Y, Z)\|^{2} \leq C_{1}|t-s|\|\eta\|_{1}^{2} \text { for } \eta \in H^{1} .
\end{gathered}
$$

For $\Phi \in \operatorname{LUC}(H)$ (= uniformly continuous functions on $H$ with linear growth), we put

$$
\tilde{\Phi}(t, \eta ; Y, Z)=E \Phi(\xi(t, \eta, Y, Z)) .
$$

Then Proposition 3.1 implies the following one. 
PROPOSITION 3.2.

(i) For $\varepsilon>0$, there is $\tau_{0}=\tau_{0}(\varepsilon, \eta, \Phi)>0$, such that for $\mathscr{Y} \in \mathcal{M}$ and $Z \in \mathcal{N}$

$$
\tilde{\Phi}(t, \eta, Y, Z)-\tilde{\Phi}(s, \eta, Y, Z) \mid<\varepsilon, \quad \text { if }|t-s|<\tau_{0} .
$$

(ii) For $\varepsilon>0$, there is $\delta_{0}=\delta_{0}(\varepsilon, \Phi)>0$, such that for $Y \in \mathcal{M}, Z \in \mathcal{N}$ and $t \in[0, T]$

$$
\left|\tilde{\Phi}(t, \eta, Y, Z)-\tilde{\Phi}\left(t, \eta^{\prime}, Y, Z\right)\right|<\varepsilon, \quad \text { if }\left\|\eta-\eta^{\prime}\right\|<\delta_{0} .
$$

Proposition 3.3. Suppose that, for any bounded set $B$ of $H, \Phi_{n} \rightarrow \Phi$ uniformly in $B$ and $\sup _{n} \sup _{\zeta \in B}\left|\Phi_{n}(\zeta)\right|<\infty$. Then, for any bounded set $B \subset H$, $\tilde{\Phi}_{n}$ tends to $\tilde{\Phi}$ uniformly in $[0, T] \times B \times \mathcal{M} \times \mathcal{N}$.

Proof. Recalling (2.3), we have

$$
P(\|\xi(t, \eta, Y, Z)\|>r) \leq K\|\eta\|^{2} / r^{2} .
$$

Now taking $r=r(\varepsilon, B)=K(\operatorname{diam} B)^{2} \varepsilon^{-2}$ and $N=N(\varepsilon, B)$ such that

$$
\begin{gathered}
\sup _{n \geq N}\left|\Phi_{n}-\Phi\right|<\varepsilon \text { on }\{\eta \in H ;\|\eta\| \leq r\}, \text { we get } \\
\quad\left|\tilde{\Phi}_{n}(t, \eta, Y, Z)-\tilde{\Phi}(t, \eta, Y, Z)\right| \\
\left\langle\varepsilon+2 \beta P\left(\|\xi(t, \eta, Y, Z)\|^{2}\right\rangle r\right)<\varepsilon+2 \varepsilon \beta
\end{gathered}
$$

where $\sup \left|\Phi_{n}(\zeta)\right|<\beta$ on $B$. This completes the proof.

Here we call $\varphi: H \rightarrow R^{1}$, a tame function, if $\varphi$ is denoted by

$$
\varphi(\eta)=\hat{\varphi}\left(\left(e_{1}, \eta\right), \cdots,\left(e_{\jmath}, \eta\right)\right)
$$

with suitable $e_{\imath} \in H^{2}$ and $\hat{\varphi} \in C^{2}\left(R^{j}\right)$ whose first and second derivatives are BUC ( = bounded and uniformly continuous function). So, $\varphi$ is Lipshitz continuous and linear growth, say

$$
|\varphi(\eta)| \leq k_{1}+k_{2}\|\eta\| \text { with } k_{\imath}=k_{i}(\hat{\varphi}) .
$$

Putting $\tilde{\varphi}(t, \eta, Y, Z)=E \varphi(\xi(t, \eta, Y, Z))$, we have

Proposition 3.4. There is a constant $C_{2}(\varphi)$ such that

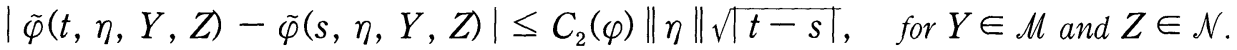


Proof. $l(\varphi)$ denotes Lipshitz constant of $\hat{\varphi}$ of (3.23).

$$
\begin{aligned}
& |E \varphi(\xi(t, \eta, Y, Z))-E \varphi(\xi(s, \eta, Y, Z))| \\
& \quad \leq l(\varphi) \sum_{i=1}^{\prime} E\left|\left(\xi(t, \eta, Y, Z)-\xi(s, \eta, Y, Z), e_{i}\right)\right| \\
& \quad \leq l(\varphi) \sum_{i=1}^{\prime} E\|\xi(t, \eta, Y, Z)-\xi(s, \eta, Y, Z)\|_{-1}\left\|e_{\imath}\right\|_{1} .
\end{aligned}
$$

So, Proposition 3.1 concludes the proof.

\section{Tame terminal function}

In this section, we deal with pay-off tame terminal function and show some compactness of value functions, employing semi-discretization arguments.

Suppose $h ; H \times \mathscr{Y} \times \mathscr{Z} \rightarrow R^{1}$ is continuous and satisfies (A5),

(A5) $h(\cdot, y, z)$ is tame, say $h(\eta, y, z)=\mathfrak{h}\left(\left(\eta, e_{1}\right), \cdots,\left(\eta, e_{q}\right), y, z\right)$ and $\left|\partial_{\imath} \mathfrak{h}(x, y, z)\right|$ and $\left|\partial_{\imath} \partial_{j} \mathfrak{h}(x, y, z)\right|$ are bounded in $(x, y, z) \in R^{q} \times \mathscr{Y} \times \mathscr{Z}$. Let $\varphi ; H \rightarrow R^{1}$ be a tame function, say

$$
\varphi(\eta)=\hat{\varphi}\left(\left(\eta, \hat{e}_{1}\right), \cdots,\left(\eta, \hat{e}_{p}\right)\right)
$$

Since we may assume that $\left\{\hat{e}_{i}, i=1, \cdots, p\right\}$ contains $\left\{e_{i}, i=1, \cdots, q\right\}$, we will

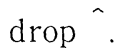

Now we define pay-off $J$ and value $V_{\hat{\mu} \hat{N}}$ as follows.

$$
\left\{\begin{aligned}
J(t, \eta, Y, Z, \varphi)=E \int_{0}^{t} h(\xi(\theta, \eta, Y, Z), Y(\theta), Z(\theta)) d \theta & \quad+\varphi(\xi(t, \eta, Y, Z)) \\
& \quad \text { for } \hat{M} \subset \mathcal{M}, \hat{\mathcal{N}} \subset \mathcal{N} .
\end{aligned}\right.
$$

Since $|h(\eta, y, z)| \leq m_{1}+m_{2}\|\eta\|$ with $m_{1}$ and $m_{2}$, which are independent of $y$ and $z,(2.3)$ derives

$$
|J(t, \eta, Y, Z, \varphi)| \leq m_{1} t+k_{1}+K\left(m_{2}+k_{2}\right)\|\eta\|
$$

whenever $|\varphi(\eta)| \leq k_{1}+k_{2}\|\eta\|$.

Appealing to Proposition 3.4, Theorem 2.2 and (2.6), we can take a positive numbers $C_{3}=C_{3}(\varphi)$ such that 


$$
\left\{\begin{array}{l}
|J(t, \eta, Y, Z, \varphi)-J(s, \zeta, Y, Z, \varphi)| \\
\quad \leq C_{3}[(1+\|\eta\|) \sqrt{|t-s|}+\|\eta-\zeta\|] \text { for } Y \in \mathcal{M} \text { and } Z \in \mathcal{N}, \\
\left|V_{\hat{M} \hat{\mathcal{N}}}(t, \eta, \varphi)-V_{\hat{\mathcal{M}} \hat{\mathcal{N}}}(s, \zeta, \varphi)\right| \leq C_{3}[(1+\|\eta\|) \sqrt{|t-s|}+\|\eta-\zeta\|] \\
\quad \text { for } \hat{M} \subset \mathcal{M} \text { and } \hat{\mathcal{N}} \subset \mathcal{N},
\end{array}\right.
$$

and, putting $S(R)=\left\{|x| \geq \frac{R}{2}\right\}$ and $\varepsilon_{R}=\sum_{t=1}^{p} \int_{S(R)} e_{\imath}^{2}(x) d x$, we have

$$
\begin{aligned}
& \left|V_{\hat{M} \hat{\mathcal{N}}}(t, \eta, \varphi)-V_{\hat{\mu} \hat{\mathcal{N}}}(s, \zeta, \varphi)\right| \\
\leq & C_{3}\left[(1+\|\eta\|) \sqrt{|t-s|}+\left\|\left(1-\zeta_{R}\right)(\eta-\zeta)\right\|_{-2}+\left(\varepsilon_{R}+\frac{1}{R}\right)\|\eta-\zeta\|\right]
\end{aligned}
$$

with $\zeta_{R}$ of Theorem 2.2. Moreover (4.4) implies

$$
V_{\hat{\mu} \hat{N}}(, \varphi) \in C\left([0, T] \times H_{w}\right)
$$

where $H_{w}$ denotes the space $H$ carrying the weak topology.

Let $\pi=\left\{0=t_{0}<t_{1}<\cdots<t_{N}=T\right\}$ be a partition on $[0, T]$ and denote $\|\pi\|=\max _{i}\left(t_{i}-t_{i-1}\right)$ its mesh. We put $\mathscr{P}(\pi)=\left\{t_{i}, i=0, \cdots, N\right\}$.

Definition 4.1. $Y \in \mathcal{M}$ (resp. $Z \in \mathcal{N}$ ) is called $\pi$-admissible for player I (resp. II), if

$$
Y(t)=Y\left(t_{j}\right)\left(\operatorname{resp} . Z(t)=Z\left(t_{j}\right)\right), \text { for } t \in\left[t_{j}, t_{j+1}\right) .
$$

$\mathcal{M}_{\pi}$ (resp. $\mathcal{N}_{\pi}$ ) denotes the set of all $\pi$-admissible controls for player I (resp. II).

Definition 4.2. $\alpha \in \Gamma$ is called a $\pi$-admissible strategy for player I, if $\alpha ; \mathcal{N}$ $\rightarrow \mathcal{M}_{\pi}$ such that

(i ) $\alpha(Z)(s)=Z$-independent for $s<t_{1}$,

(ii) $\alpha(Z)\left(t_{j}\right)=\alpha(\hat{Z})\left(t_{j}\right)$ a.e., if $Z=\tilde{Z}$ a.e. on $\left[0, t_{j}\right)$.

$\beta \in \Delta$ is called a $\pi$-admissible strategy for player II, if $\beta ; \mathcal{M} \rightarrow \mathcal{N}_{\pi}$ such that

(i ) $\beta(Y)(s)=Y$-independent for $s<t_{1}$,

(ii) $\beta(Y)\left(t_{j}\right)=\beta(\hat{Y})\left(t_{j}\right)$ a.e., if $Y=\tilde{Y}$ a.e. on $\left[0, t_{j}\right]$.

$\Gamma_{\pi}$ (resp. $\Delta_{\pi}$ ) denotes the set of all $\pi$-admissible strategies for player I (resp. II).

Let us set

$$
J(t, \eta, Y, \beta, \varphi)=J(t, \eta, Y, \beta(Y), \varphi)
$$

and the upper value function $V_{\pi}$ for $\pi$ is defined by (4.6) below,

$$
V_{\pi}(t, \eta, \varphi)=\inf _{\beta \in \Delta_{\pi}} \sup _{Y \in \mathcal{M}} J(t, \eta, Y, \beta, \varphi) .
$$


From (4.3) and (4.4), we can easily see

Proposition 4.1. $V_{\pi}(, \varphi)$ has $\pi$-independent continuity.

In particular, (4.4) implies compactness of upper value functions, namely, we have the following theorem.

THEOREM 4.1. Let $\pi_{n}, n=1,2, \cdots$, be a sequence of partitions on $[0, T]$. Then, $V_{\pi_{n}}(, \varphi), n=1,2, \cdots$, has a sequence which converges uniformly in any bounded set of $[0, T] \times H$.

For the proof, we will show the following lemma.

Lemma. Let $\varepsilon>0$ and $B=\{\eta \in H ;\|\eta\| \leq r\}$. Then we can take a finite set $D \subset[0, T] \times B$, such that for $(t, \eta) \in[0, T] \times B$ there is $(s, \zeta) \in D$ satisfying

$$
\left|V_{\pi}(t, \eta, \varphi)-V_{\pi}(s, \zeta, \varphi)\right|<\varepsilon, \text { for any partition } \pi \text {. }
$$

Proof. Let us take a large number $R=R(r, \varepsilon)$, such that

$$
C_{3}(\varphi)\left(\varepsilon_{R}+\frac{1}{R}\right)(2 r)<\varepsilon
$$

So we may assume $R(r, \varepsilon) \rightarrow \infty$, as $\varepsilon \rightarrow 0$. Denote $S=\left\{x \in R^{n} ;|x| \leq R\right)$ and $\hat{\zeta}=\zeta_{R}$ of Theorem 2.2. Then supp $(1-\hat{\zeta}) \eta \subset S$. Since $B$ is weakly compact in $H,\{(1-\hat{\zeta}) \eta, \eta \in B\}$ is compact in $H^{-2}(S)$. Hence we can take a finite set $E \subset$ $B$, such that $\{(1-\hat{\zeta}) \eta, \eta \in E\}$ is $\varepsilon\left(3 C_{3}(\varphi)\right)^{-1}$-net in $H^{-2}(S)$.

Let $\Theta$ be a finite $\left(3 C_{3}(\varphi)(1+r)\right)^{-2} \varepsilon^{2}$-net in $[0, T]$. Then (4.4) asserts that, for $(t, \eta) \in[0, T] \times B$, there is $(s, \zeta) \in \Theta \times E$ such that

$$
\left|V_{\pi}(t, \eta, \varphi)-V_{\pi}(s, \zeta, \varphi)\right|<\varepsilon, \text { for any } \pi \text {. }
$$

This completes the proof of Lemma.

Proof of Theorem. Denote $D$ of Lemma by $D(r, \varepsilon)$ and put $\mathscr{D}=\bigcup_{r, k=1}^{\infty} D\left(r, \frac{1}{k}\right)$. Since $\left|V_{\pi}(, \varphi)\right|_{\infty} \leq m_{1} T+k_{1}(\varphi)+K\left(m_{2}+k_{2}(\varphi)\right)\|\eta\|$, some subsequence $V_{\pi_{n}},(s, \zeta, \varphi)$ converges for any $(s, \zeta) \in \mathscr{D}$. Now

Lemma implies Theorem.

Put $V(, \varphi)=\lim V_{\pi_{n}}(, \varphi)$. Then $V$ also satisfies (4.3) and (4.4). 


\section{Uniformly continuous terminal function with linear growth}

Since an upper value function $V_{\pi}(\cdot, \varphi)$ may not be tame, we will study pay-off with continuous terminal function, in order to prove the principle of dynamic programming in Section 6.

It is clear that $J(t, \cdot, Y, Z, \Phi) \in \operatorname{LUC}(H)$ whenever $\Phi \in \operatorname{LUC}(H)$. Moreover we can easily see the following proposition.

PROPOSITION 5.1.

(i ) For $\varepsilon>0$, there is $\delta_{0}=\delta_{0}(\varepsilon, \Phi)>0$, such that

$$
|J(t, \eta, Y, Z, \Phi)-J(t, \hat{\eta}, Y, Z, \Phi)|<\varepsilon
$$

and

$$
\left|V_{\hat{M} \hat{N}}(t, \eta, \Phi)-V_{\hat{M} \hat{N}}(t, \hat{\eta}, \Phi)\right|<\varepsilon
$$

whenever $\|\eta-\hat{\eta}\|<\delta_{0}$.

(ii) For $\varepsilon>0$, there is $\hat{\delta}_{0}=\delta_{0}(\varepsilon, \phi, \eta)>0$, such that

$$
\left|V_{\widehat{M} \hat{\mathcal{N}}}(t, \eta, \Phi)-V_{\widehat{M} \hat{\mathcal{N}}}(s, \eta, \Phi)\right|<\varepsilon \text {, whenever }|t-s|<\hat{\delta}_{0} .
$$

Let us set

$$
\left\{\begin{array}{l}
v(t, \eta, z, \Phi)=\sup _{Y \in \mathcal{M}} J(t, \eta, Y, z, \Phi) \\
v(t, \eta, \Phi)=\inf _{z \in \mathscr{Z}} v(t, \eta, z, \Phi)
\end{array}\right.
$$

Then (5.2) implies that $v(t, \quad, z, \Phi) \in \mathrm{LUC}(H)$ and

$$
|v(t, \eta, z, \Phi)-v(t, \hat{\eta}, z, \Phi)|<\varepsilon \text { for any }(t, z) \in[0, T] \times \mathscr{Z},
$$

whenever $\|\eta-\hat{\eta}\|<\delta_{0}$.

First we study the continuity of $v$ with respect to $z$. Putting

$$
\hat{\xi}(t)=\xi(t, \eta, Y, \hat{z}) \text { and } \zeta(t)=\xi(t, \eta, Y, z)-\hat{\xi}(t)
$$

we can see

$\left\{\begin{array}{l}d \zeta(t)=[L(Y(t), z)-c I \zeta(t) d t+f(Y(t), z) \zeta(t) d W(t)+F(t) d t+G(t) d W(t) \\ \zeta(0)=0\end{array}\right.$

where $F(t)=[L(Y(t, z)-L(Y(t), \hat{z})] \hat{\xi}(t)$ and $G(t)=[f(Y(t, z)-f(Y(t), \hat{z})] \hat{\xi}(t)$. 
Employing the standard arguments, we obtain the following one.

Proposition 5.2.

(i) There is a constant $C_{4}$ independent of $Y, z$ and $\eta$ such that

$$
E\left(\sup _{t \leq T}\|\xi(t, \eta, Y, z)-\xi(t, \eta, Y, \hat{z})\|^{2}\right) \leq C_{4}\|\eta\|^{2}|z-\hat{z}|^{2} .
$$

(ii) For $\varepsilon>0$ and $k$, there is $\delta_{k}=\delta_{k}(\varepsilon, \Phi)>0$, such that

$$
\begin{aligned}
& |J(t, \eta, Y, z, \Phi)-J(t, \eta, Y, \hat{z}, \Phi)|<\varepsilon, \\
& \quad \text { for } t \in[0, T], Y \in M, \eta \in B(k)=\{\eta \| \leq k\},
\end{aligned}
$$

whenever $|z-\hat{z}|<\delta_{k}$.

(iii) $v(t, \eta, z, \Phi)$ satisfies (5.7).

Now we will prove the principle of dynamic programming, applying the similar arguments as [4]. Let $\mathfrak{P}_{\varepsilon}^{H}\left(=\mathfrak{B}_{\varepsilon \Phi}^{H}\right)=\{\mathscr{E}, j=1,2, \cdots\}$ be a Borel partition of $H$, with $\operatorname{diam}_{\mathscr{E}_{j}}<\delta_{0}(\varepsilon, \Phi)$ (see Proposition 5.1 for $\delta_{0}$ ). For simplicity, we may assume $\mathscr{E}_{j} \subset B(j)$. Since $\mathscr{Z}$ is compact, for any fixed $\eta_{j} \in \mathscr{E}_{j}, v\left(t, \eta_{j}, \cdot, \Phi\right)$ has a minimum point $z_{j}^{*}=z^{*}\left(t, \eta_{j}, \Phi\right)$, namely

$$
v\left(t, \eta_{j}, z_{j}^{*}, \Phi\right)=\boldsymbol{v}\left(t, \eta_{j}, \Phi\right) .
$$

Taking an approximate maximum point $Y_{j}^{*}=Y_{j}^{*}\left(t, \eta_{j}, \Phi, \varepsilon\right)$ below,

$$
v\left(t, \eta_{j}, z_{j}^{*}, \Phi\right)-\varepsilon<J\left(t, \eta_{j}, Y_{j}^{*}, z_{j}^{*}, \Phi\right) \leq v\left(t, \eta_{j}, z_{j}^{*}, \Phi\right)
$$

we have

$$
0 \leq \boldsymbol{v}\left(t, \eta_{j}, \Phi\right)-J\left(t, \eta_{j}, Y_{j}^{*}, z_{j}^{*}, \Phi\right)<\varepsilon .
$$

Hence Proposition 5.2 asserts

$$
\left|\boldsymbol{v}(t, \eta, \Phi)-J\left(t, \eta, Y_{j}^{*}, z_{j}^{*}, \Phi\right)\right|<3 \varepsilon, \text { for } \eta \in \mathscr{E}_{j} .
$$

Appealing to $J\left(t, \eta_{j}, Y, z_{j}^{*}, \Phi\right) \leq v\left(t, \eta_{j}, z_{j}^{*}, \Phi\right)$ for $Y \in \mathcal{M}$, we get

$$
J\left(t, \eta, Y, z_{j}^{*}, \Phi\right) \leq v(t, \eta, \Phi)+2 \varepsilon .
$$

For $\pi=\left\{0=t_{0}<t_{1}<\cdots<t_{N}=T\right\}$ and $\tau \in\left(t_{p-1}, t_{p}\right]$, we will define $\boldsymbol{v}(t) ; \operatorname{LUC}(H) \rightarrow \operatorname{LUC}(H)$ and $\Phi_{j}\left(=\Phi_{j}^{\tau}\right)$ as follows

$$
\begin{aligned}
& \boldsymbol{v}(t) \Phi=\boldsymbol{v}(t, \cdot, \Phi) \\
& \Phi_{p}=\Phi, \quad \Phi_{p-1}=v\left(\tau-t_{p-1}\right) \Phi_{p}, \Phi_{p-2}=v\left(s_{p-1}\right) \Phi_{p-1}, \cdots, \Phi_{0}=v\left(s_{1}\right) \Phi_{1}
\end{aligned}
$$


where $s_{j}=t_{j}-t_{j-1}$. Appealing to (5.8), we will choose an approximately optimal strategy $\beta_{\varepsilon} \in \Delta_{\pi}$ in the following way. Fixing $Y \in \mathcal{M}$ and $\eta_{j, k} \in \mathscr{E}_{j}\left(\in \mathfrak{B}_{\varepsilon \Phi_{k}}^{H}\right)$ arbitrarily, $\beta_{\varepsilon}(Y)$ is defined by

$$
\begin{aligned}
\beta_{\varepsilon}(Y)(\theta) & =I_{\left[0, t_{1}\right)}(\theta) \sum_{\left(\Phi_{1}\right)} I_{\mathscr{G},}(\eta) z^{*}\left(s_{1}, \eta_{j, 1}, \Phi_{1}\right) \\
& +I_{\left[t_{1}, t_{2}\right)}(\theta) \sum_{\left(\Phi_{2}\right)} I_{\mathscr{E},}\left(\zeta\left(t_{1}, \eta\right)\right) z^{*}\left(s_{2}, \eta_{j, 2}, \Phi_{2}\right)+\cdots \\
& +I_{\left[t_{p-1}, T\right)}(\theta) \sum_{\left(\Phi_{p}\right)} I_{\mathscr{E},}\left(\zeta\left(t_{p-1}, \eta\right)\right) z^{*}\left(\tau-t_{p-1}, \eta_{j, p}, \Phi_{p}\right)
\end{aligned}
$$

where $I_{A}=$ indicator function of $A, \sum_{(\Phi)}=\sum_{\mathscr{E}_{1} \in \mathfrak{F}_{\varepsilon \Phi}^{H} \Phi}$ and $\zeta(t, \eta)$ is a solution of (2.1) for $\left(Y, \beta_{\varepsilon}(Y)\right)$ with $\zeta(0)=\eta$, namely,

$$
\begin{aligned}
& \zeta(t, \eta)=\sum_{\left(\Phi_{1}\right)} I_{\mathscr{E},}(\eta) \xi\left(t, \eta, Y, z^{*}\left(s_{1}, \eta_{j, 1}, \Phi_{1}\right)=\xi\left(t, \eta, Y, \beta_{\varepsilon}(Y)\right),\right. \\
& \text { for } t \leq t_{1} \text {, }
\end{aligned}
$$

Hence $\beta_{\varepsilon}(Y)$ on $\left[t_{1}, t_{2}\right)$ is determined by (5.14) and $\zeta(\theta, \eta)$ by $\xi(\theta, \eta, Y$, $\left.\beta_{\varepsilon}(Y)\right)$ for $\theta \in\left[0, t_{2}\right]$. Repeating the same arguments, we can determine $\beta_{\varepsilon}(Y)$ of (5.14) and

$$
\zeta(t, \eta)=\xi\left(t, \eta, Y, \beta_{\varepsilon}(Y)\right) \text { for } t \in[0, T] .
$$

Since $\beta_{\varepsilon}(Y)(\theta)$ is $\mathscr{F}_{t_{p-1}}$-measurable and $Y(\theta)$ is a Borel function of $\{W(t), t$ $\left.\leq t_{p-1}\right\}$ and $\left\{W(s)-W\left(t_{p-1}\right), t_{p-1} \leq s \leq \theta\right\}$, putting $\xi(t)=\xi\left(t, \eta, Y, \beta_{\varepsilon}\right)$, we have

$$
\begin{aligned}
& E\left(\int_{t_{p-1}}^{\tau} h\left(\xi(\theta), Y(\theta), \beta_{\varepsilon}(Y)(\theta)\right) d \theta+\Phi(\xi(\tau)) / \mathscr{F}_{t_{p-1}}\right) \\
& \quad \leq \boldsymbol{v}\left(\tau-t_{p-1}, \xi\left(t_{p-1}\right), \Phi\right)+2 \varepsilon, \text { a.s. }
\end{aligned}
$$

by virtue of (5.12). Thus, it follows that

$$
\begin{aligned}
& J\left(\tau, \eta, Y, \beta_{\varepsilon}, \Phi\right) \\
& \leq E\left[\int_{0}^{t_{p-1}} h\left(\xi(\theta), Y(\theta), \beta_{\varepsilon}(Y)(\theta)\right) d \theta+v\left(\tau-t_{p-1}\right) \Phi\left(\xi\left(t_{p-1}\right)\right)+2 \varepsilon\right] \\
& =E\left[\int_{0}^{t_{p-1}} h\left(\xi(\theta), Y(\theta), \beta_{\varepsilon}(Y)(\theta)\right) d \theta+\Phi_{p-1}\left(\xi\left(t_{p-1}\right)\right)+2 \varepsilon\right] .
\end{aligned}
$$

Repeating the same arguments, we obtain

$$
J\left(\tau, \eta, Y, \beta_{\varepsilon}, \Phi\right) \leq v\left(s_{1}\right) v\left(s_{2}\right) \cdots v\left(\tau-t_{p-1}\right) \Phi(\eta)+2 p \varepsilon .
$$

Since $Y$ is arbitrary, it follows that 


$$
\sup _{Y \in \mathscr{M}} J\left(\tau, \eta, Y, \beta_{\varepsilon}, \Phi\right) \leq \boldsymbol{v}\left(s_{1}\right) \boldsymbol{v}\left(s_{2}\right) \cdots v\left(\tau-t_{p-1}\right) \Phi(\eta)+2 p \varepsilon .
$$

From the fact $\beta_{\varepsilon} \in \Delta_{\pi}$, we see, letting $\varepsilon \rightarrow 0$,

$$
\inf _{\beta \in \Delta_{\pi}} \sup _{Y \in \mathscr{M}} J(\tau, \eta, Y, \beta, \Phi) \leq \boldsymbol{v}\left(s_{1}\right) \boldsymbol{v}\left(s_{2}\right) \cdots v\left(\tau-t_{p-1}\right) \Phi(\eta) .
$$

Now we will choose nearly optimal $\alpha_{\varepsilon} \in \Gamma$ in the following way. Fix $\eta_{j} \in \mathscr{E}_{j}$ $\in \mathfrak{P}_{\varepsilon \Phi}^{H}$ arbitrarily. Noting the compactness of $\mathscr{Z}$, let us denote $\mathfrak{B}_{\varepsilon j \Phi}^{\mathscr{Z}}=\left\{S_{1}, \cdots, S_{m}\right\}$ a finite partition of $\mathscr{L}$, with $\operatorname{diam} S_{\imath}<\delta_{j}$, (see (5.7)). For any $z_{i} \in S_{\imath} \in \mathfrak{B}_{\varepsilon j \Phi}^{\mathscr{Z}}$, we will take $Y^{*}=Y^{*}\left(t, \eta_{\jmath}, z_{j}, \Phi\right) \in \mathcal{M}$ such that

$$
J\left(t, \eta_{\jmath}, Y^{*}, z_{\imath}, \Phi\right)>v\left(t, \eta_{j}, z_{\imath}, \Phi\right)-\varepsilon .
$$

Then it follows from (5.1) and (5.7) that

$$
J\left(t, \eta, Y^{*}, z_{\imath}, \Phi\right)>v\left(t, \eta, z_{\imath}, \Phi\right)-3 \varepsilon \text { for } \eta \in \mathscr{E}_{j}
$$

and

$$
J\left(t, \eta, Y^{*}, z, \Phi\right)>v(t, \eta, z, \Phi)-5 \varepsilon \quad \text { for } \eta \in \mathscr{E}_{j} \text { and } z \in S_{i} .
$$

When we stress the dependence on Brownian path $w$, we will denote $Y(\theta)$ by $Y(\theta, w)$. Putting $Y_{p, j, i}^{*}=Y^{*}\left(\tau-t_{p-1}, \eta_{j}, z_{\imath}, \Phi_{p}\right)$ and $Y_{k, j, l}^{*}=Y^{*}\left(t_{k}-t_{k-1}, \eta_{j}\right.$, $\left.z_{i}, \Phi_{k}\right)$ for $\eta_{j} \in \mathscr{E}_{j} \in \mathfrak{B}_{\varepsilon \Phi_{k}}^{H}$ and $z_{\imath} \in S_{\imath} \in \mathfrak{P}_{\varepsilon j \Phi_{k}}^{\mathscr{Z}}, k=p-1, \cdots, 0$, we define $\alpha_{\varepsilon}(Z)$ as follows,

$$
\begin{aligned}
& \alpha_{\varepsilon}(Z)(\theta, w)=I_{\left[0, t_{1}\right)}(\theta) \sum_{\left(\Phi_{1}\right)}^{*} I_{\mathscr{E}_{1}}(\eta) I_{S_{i}}(Z(0, w)) Y_{1, j, 2}^{*}(\theta, w) \\
& +I_{\left[t_{1}, t_{2}\right)}(\theta) \sum_{\left(\Phi_{2}\right)}^{*} I_{\mathscr{E}_{1}}\left(\zeta\left(t_{1}, \eta\right)\right) I_{S_{i}}\left(Z\left(t_{1}, w\right)\right) Y_{2, j, 2}^{*}\left(\theta-t_{1}, w_{t_{1}}^{+}\right) \\
& \quad+\cdots \\
& +I_{\left[t_{p-1}, T\right]}(\theta) \sum_{\left(\Phi_{p}\right)}^{*} I_{\mathscr{E}_{j}}\left(\zeta\left(t_{p-1}, \eta\right)\right) I_{S_{i}}\left(Z\left(t_{p-1}, w\right)\right) Y_{p, j, l}^{*}\left(\theta-t_{p-1}, w_{t_{p-1}}^{+}\right),
\end{aligned}
$$

where $\sum_{(\Phi)}^{*}=\sum_{\delta, \in \Re_{s o}^{H}} \sum_{S_{t} \in \Re_{\varepsilon, \theta}^{*}}, w_{t}^{+}$is a shifted path, namely $w_{t}^{+}(\theta)=w(t+\theta)-$ $w(t)$, and $\zeta(t, \eta)=\xi\left(t, \eta, \alpha_{\varepsilon}(Z), Z\right)$. Applying the same arguments as (5.16) (5.18), we obtain

$$
\begin{array}{r}
J\left(\tau, \eta, \alpha_{\varepsilon}, Z, \Phi\right) \geq \boldsymbol{v}\left(s_{1}\right) \boldsymbol{v}\left(s_{2}\right) \cdots \boldsymbol{v}\left(\tau-t_{p-1}\right) \Phi(\eta)-5 p \varepsilon \\
\text { for } Z \in \mathcal{N}_{\pi} .
\end{array}
$$

Thus it follows that

$$
\inf _{Z \in \mathcal{N}_{\pi}} J\left(\tau, \eta, \alpha_{\varepsilon}, Z, \Phi\right) \geq \boldsymbol{v}\left(s_{1}\right) \boldsymbol{v}\left(s_{2}\right) \cdots v\left(\tau-t_{p-1}\right) \Phi(\eta)-5 p \varepsilon
$$

Since $\alpha_{\varepsilon} \in \Gamma$, letting $\varepsilon \rightarrow 0$, we get 


$$
\sup _{\alpha \in \Gamma} \inf _{Z \in \mathcal{N}_{\pi}} J(\tau, \eta, \alpha, Z, \Phi) \geq \boldsymbol{v}\left(s_{1}\right) \boldsymbol{v}\left(s_{2}\right) \cdots v\left(\tau-t_{p-1}\right) \Phi(\eta) .
$$

Combining with (5.20), we have Proposition 5.3 below,

Proposition 5.3. Let $\Phi \in \operatorname{LUC}(H), \mathscr{P}_{\pi}=\left\{t_{j}, j=0, \cdots, N\right\}$ and $\tau \in\left(t_{p-1}, t_{p}\right]$. For $\varepsilon>0$, there exist $\alpha_{\varepsilon} \in \Gamma$ and $\beta_{\varepsilon} \in \Delta_{\pi}$ such that, for $Y \in M$ and $Z \in \mathcal{N}_{\pi}$,

$$
\begin{aligned}
J\left(\tau, \eta, Y, \beta_{\varepsilon}, \Phi\right)-\varepsilon & <v\left(t_{1}\right) v\left(t_{2}-t_{1}\right) \cdots v\left(\tau-t_{p-1}\right) \Phi(\eta) \\
& <J\left(\tau, \eta, \alpha_{\varepsilon}, Z, \Phi\right)+\varepsilon, \quad \text { for } \eta \in H .
\end{aligned}
$$

Moreover, according to [4], we obtain

Proposition 5.4 .

$$
\begin{aligned}
& \inf _{\beta \in \Delta_{\pi}} \sup _{Y \in \mathcal{M}} J(\tau, \eta, Y, \beta, \Phi)=\boldsymbol{v}\left(t_{1}\right) \boldsymbol{v}\left(t_{2}-t_{1}\right) \cdots v\left(\tau-t_{p-1}\right) \Phi(\eta) \\
& =\sup _{\alpha \in \Gamma} \inf _{Z \in \mathcal{N}_{\pi}} J(\tau, \eta, \alpha, Z, \Phi) .
\end{aligned}
$$

For the proof, we will recall the following lemma.

Lemma [4]. For any $\beta \in \Delta_{\pi}$ and $\alpha \in \Gamma$, there exist $Y^{*} \in \mathcal{M}$ and $Z^{*} \in \mathcal{N}_{\pi}$ such that

$$
J\left(\tau, \eta, \alpha, Z^{*}, \Phi\right)=J\left(\tau, \eta, Y^{*}, \beta, \Phi\right) .
$$

Outline of proof. For any fixed $z_{0} \in \mathscr{Z}$, let us define $Y_{k} \in \mathcal{M}$ and $z_{k} \in \mathcal{N}_{\pi}$, as follows.

$$
\begin{gathered}
Y_{0}(s)=\alpha\left(z_{0}\right)(s), Z_{0}(s)=\beta\left(Y_{0}\right)(s), Y_{1}(s)=\alpha\left(Z_{0}\right)(s), \\
Z_{1}(s)=\beta\left(Y_{1}\right)(s), \cdots, Y_{k}(s)=\alpha\left(Z_{k-1}\right)(s), Z_{k}(s)=\beta\left(Y_{k}\right)(s), \cdots
\end{gathered}
$$

Then we have

$$
Y_{j+1}=Y_{j} \text { on }\left[0, t_{j}\right] \text { and } Z_{j+1}=Z_{j} \text { on }\left[0, t_{j}\right) \text {. }
$$

In fact, $\beta(Y)(s), s<t_{1}$, is independent of $Y$, since $\beta \in \Delta_{\pi}$. Hence it follows that $\beta\left(Y_{0}\right)=\beta\left(Y_{1}\right)=\cdots=\beta\left(Y_{k}\right)$ on $\left[0, t_{1}\right)$, and

$$
Z_{0}=Z_{1}=\cdots=Z_{k} \text { on }\left[0, t_{1}\right) .
$$

Now (ii) of Definition (4.2) yields 


$$
\alpha\left(Z_{0}\right)\left(t_{1}\right)=\alpha\left(Z_{1}\right)\left(t_{1}\right)=\cdots=\alpha\left(Z_{k}\right)\left(t_{1}\right)
$$

and

$$
\alpha\left(Z_{0}\right)=\alpha\left(Z_{1}\right)=\cdots=\alpha\left(Z_{k}\right) \text { on }\left[0, t_{1}\right)
$$

This asserts

$$
Y_{1}=Y_{2}=\cdots=Y_{k+1} \text { on }\left[0, t_{1}\right] \text {. }
$$

So $\beta\left(Y_{1}\right)\left(t_{1}\right)=\cdots=\beta\left(Y_{k+1}\right)\left(t_{1}\right)$. Since $\beta \in \Delta_{\pi}$, we see

$$
\beta\left(Y_{1}\right)=\cdots=\beta\left(Y_{k+1}\right) \text { on }\left[t_{1}, t_{2}\right) .
$$

From (5.32) and (5.34), we can see

$$
Z_{1}=\cdots=Z_{k+1} \text { on }\left[0, t_{2}\right) .
$$

Repeating the same arguments, we can conclude (5.31).

Putting $Y^{*}=Y_{N}$ and $Z^{*}=Z_{N-1}$, we get

$$
\alpha\left(Z^{*}\right)=Y^{*} \text { and } \beta\left(Y^{*}\right)=Z^{*} \text {. }
$$

Now the proof is completed.

Proof of Proposition 5.4. From (5.28), we can easily see

$$
\begin{gathered}
\inf _{\beta \in \Delta_{\pi}} \sup _{Y \in \mathscr{M}} J(\tau, \eta, Y, \beta, \Phi) \leq \boldsymbol{v}\left(t_{1}\right) \boldsymbol{v}\left(t_{2}-t_{1}\right) \cdots v\left(\tau-t_{p-1}\right) \Phi(\eta) \\
\leq \sup _{\alpha \in \Gamma} \inf _{Z \in \mathcal{N}_{\pi}} J(\tau ; \eta, \alpha, Z, \Phi) .
\end{gathered}
$$

For $\varepsilon>0$, we take $\hat{\beta} \in \Delta_{\pi}$ and $\hat{\alpha} \in \Gamma$ such that

$$
\left\{\begin{array}{l}
\sup _{Y \in \mathscr{M}} J(\tau, \eta, Y, \hat{\beta}, \Phi)-\varepsilon<\inf _{\beta \in \Delta_{\pi}} \sup _{Y \in \mathscr{M}} J(\tau, \eta, Y, \beta, \Phi) \\
\inf _{Z \in \mathcal{N}_{\pi}} J(\tau, \eta, \hat{\alpha}, Z, \Phi)+\varepsilon>\sup _{\alpha \in \Gamma} \inf _{Z \in \mathcal{N}_{\pi}} J(\tau, \eta, \alpha, Z, \Phi) .
\end{array}\right.
$$

Now it follows from Lemma that

$$
\left\{\begin{array}{l}
J\left(\tau, \eta, Y^{*}, \beta, \Phi\right) \leq \sup _{Y \in \mathscr{M}} J(\tau, \eta, Y, \beta, \Phi) \\
J\left(\tau, \eta, \hat{\alpha}, Z^{*}, \Phi\right) \geq \inf _{Z \in \mathcal{N}_{\pi}} J(\tau, \eta, \hat{\alpha}, Z, \Phi) \\
J\left(\tau, \eta, Y^{*}, \beta, \Phi\right)=J\left(\tau, \eta, \hat{\alpha}, Z^{*}, \Phi\right)
\end{array}\right.
$$

holds for some $Y^{*} \in \mathcal{M}$ and $Z^{*} \in \mathcal{N}_{\pi}$. Thus, (5.37) and (5.38) complete the proof. 
For $\pi=\left\{0=t_{0}<\cdots<t_{N}=T\right\}$ and $t \in\left[t_{j}, t_{j+1}\right)$, we denote $\pi(t)=\{0$ $\left.<t_{j+1}-t<t_{j+2}-t<\cdots<t_{N}-t\right\}$. Putting

$$
V_{\pi}(\tau, \eta, \Phi)=\inf _{\beta \in \Delta_{\pi}} \sup _{Y \in \mathscr{M}} J(\tau, \eta, Y, \beta, \Phi),
$$

(5.29) yields the principle of dynamic programming below,

$$
V_{\pi}(\tau, \eta, \Phi)=V_{\pi}\left(t_{j}, \eta, V_{\pi\left(t_{j}\right)}\left(\tau-t_{j}, \cdot, \Phi\right)\right) .
$$

Namely, for $0 \leq s \leq T-t_{j}$,

$$
V_{\pi}\left(s+t_{j}, \eta, \Phi\right)=V_{\pi}\left(t_{j}, \eta, V_{\pi\left(t_{j}\right)}(s, \cdot, \Phi)\right)
$$

holds.

\section{Principle of dynamic programming}

For partitions $\pi$ and $\hat{\pi}$, we say $\pi \leq \hat{\pi}$ if $\mathscr{P}_{\pi} \subset \mathscr{P}_{\hat{\pi}}$, where $\mathscr{P}_{\pi}$ denotes the set of division points of $\pi$ (see Section 4 ).

Proposition 6.1. Suppose $\pi_{n} \leq \pi_{n+1}, n=1,2 \cdots$, and put

$$
V(\tau, \eta, \Phi)=\inf _{\beta \in \cup \Delta_{\pi_{n}}} \sup _{Y \in M} J(\tau, \eta, Y, \beta, \Phi) .
$$

Then,

$$
V_{\pi_{n}}(, \Phi) \geq V_{\pi_{n+1}}(, \Phi) \text { and } V_{\pi_{n}}(\tau, \eta, \Phi) \rightarrow V(\tau, \eta, \Phi) \text { for any } \tau, \eta
$$

Proof is easy.

In particular, Theorem 4.1 implies, for a tame function $\varphi$

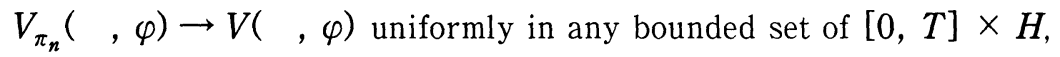

as $n \rightarrow \infty$.

Moreover we will prove

THeorem 6.1. Let $\pi_{n}<\pi_{n+1}$ and $\lim \left\|\pi_{n}\right\|=0$. Then, $V(, \Phi)$ of $(6.3)$ does not depend on a sequence $\left\{\pi_{n}\right\}$.

First we will prove the following lemma.

Lemma. Let $\mathscr{P}_{\pi}=\left\{t_{j}, j=0, \cdots, N\right\}, \mathscr{P}_{\hat{\pi}}=\mathscr{P}_{\pi} \cup\{\tau\} \quad$ and $\quad t_{p-1}<\tau<t_{p}$. Then there is a constant $k=k(\varphi)$, independent of $\pi$, such that 


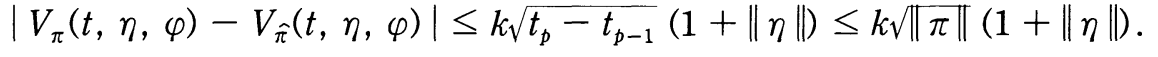

Proof of Lemma. For $t \in\left(t_{j-1}, t_{j}\right], j>p$, we put

$$
\Phi=\boldsymbol{v}\left(t_{p+1}-t_{p}\right) \boldsymbol{v}\left(t_{p+2}-t_{p+1}\right) \cdots \boldsymbol{v}\left(t-t_{j-1}\right) \varphi .
$$

Since $\pi$ and $\hat{\pi}$ have the same division points on $\left[t_{p}, t\right)$, (5.29) implies

$$
\begin{aligned}
& \Phi(\eta)=\inf _{\beta \in \Delta_{\pi}} \sup _{Y \in \mathcal{M}} J\left(t-t_{p}, \eta, Y, \beta, \varphi\right)=\inf _{\beta \in \Delta_{\hat{\pi}}} \sup _{Y \in \mathcal{M}} J\left(t-t_{p}, \eta, Y, \beta, \varphi\right), \\
& \boldsymbol{v}\left(t_{p}-t_{p+1}\right) \Phi(\eta)=\inf _{\beta \in \Delta_{\pi}} \sup _{Y \in \mathcal{M}} J\left(t-t_{p-1}, \eta, Y, \beta, \varphi\right), \quad \operatorname{say} \Phi^{*}(\eta),
\end{aligned}
$$

and

$$
\boldsymbol{v}\left(\tau-t_{p+1}\right) \boldsymbol{v}\left(t_{p}-\tau\right) \Phi(\eta)=\inf _{\beta \in \Delta_{\tilde{\pi}}} \sup _{Y \in \mathcal{M}} J\left(t-t_{p-1}, \eta, Y, \beta, \varphi\right) \text {, say } \hat{\Phi}(\eta) .
$$

Therefore, we see, from (4.3),

$$
\left|\Phi^{*}(\eta)-\Phi(\eta)\right| \leq C_{3}(\varphi) \sqrt{t_{p}-t_{p-1}}(1+\|\eta\|)
$$

and

$$
|\hat{\Phi}(\eta)-\Phi(\eta)| \leq C_{3}(\varphi) \sqrt{t_{p}-t_{p-1}}(1+\|\eta\|) .
$$

Again same arguments yield

$$
\text { 1) } \begin{aligned}
& \left|V_{\pi}(t, \eta, \varphi)-\boldsymbol{v}\left(t_{1}\right) \cdots \boldsymbol{v}\left(t_{p-1}-t_{p-2}\right) \Phi(\eta)\right| \\
= & \left|\boldsymbol{v}\left(t_{1}\right) \cdots v\left(t_{p-1}-t_{p-2}\right) \Phi^{*}(\eta)-\boldsymbol{v}\left(t_{1}\right) \cdots v\left(t_{p-1}-t_{p-2}\right) \Phi(\eta)\right| \\
\leq & \sup _{Y \in \mathcal{M}, Z \in \mathcal{N}} E\left|\Phi^{*}\left(\xi\left(t_{p-1}, \eta, Y, Z\right)\right)-\Phi\left(\xi\left(t_{p-1}, \eta, Y, Z\right)\right)\right| \\
\leq & C_{3}(\varphi) \sqrt{t_{p}-t_{p-1}}\left(1+\sup _{Y, Z} E\left\|\xi\left(t_{p-1}, \eta, Y, Z\right)\right\|\right) \leq \hat{k}(\varphi) \sqrt{t_{p}-t_{p-1}}(1+\|\eta\|)
\end{aligned}
$$

with a constant $\hat{k}(\varphi)$ by (2.3), and similarly for $\hat{\pi}$. This concludes the proof.

More generally, we obtain

$$
\left|V_{\pi}(t, \eta, \varphi)-V_{\hat{\pi}}(t, \eta, \varphi)\right| \leq k^{*}(\varphi) \sqrt{\|\pi\|(1+\|\eta\|)}
$$

with a constant $k^{*}(\varphi)$, if $\mathscr{P}_{\hat{\pi}}=\mathscr{P}_{\pi} \cup\left\{\tau_{1}, \tau_{2}, \cdots, \tau_{q}\right\}$.

Proof of Theorem 6.1. Let $\pi \vee \hat{\pi}$ be the partition with division points $\mathscr{P}_{\pi} \cup$ $\mathscr{P}_{\hat{\pi}}$. Setting $\pi_{n, k}=\pi_{\pi} \vee \hat{\pi}_{k},\left\{\pi_{n, k}, k=1,2, \cdots\right\}$ and $\left\{\pi_{n, k}, n=1,2, \cdots\right\}$ again satisfy the condition of Theorem. Put $V=\lim V_{\pi_{n}}, V_{k}=\lim _{n \rightarrow \infty} V_{\pi_{n, k}}, \hat{V}=\lim V_{\hat{\pi}_{n}}$ and $\hat{V}_{n}=\lim _{k \rightarrow \infty} V_{\pi_{n, k}}$. Then 


$$
V_{k} \leq V_{\pi_{n, k}}
$$

holds. Moreover, (6.12) implies

$$
\left|V_{\pi_{n}}(t, \eta, \varphi)-V_{\pi_{n, k}}(t, \eta, \varphi)\right| \leq \hat{k}(\varphi) \sqrt{\left\|\pi_{n}\right\|(1+\|\eta\|) .}
$$

Hence, letting $n$ to $\infty$, we get

$$
V=V_{k}, k=1,2, \cdots .
$$

In the same way, we see

$$
\hat{V}=\hat{V}_{n}, n=1,2, \cdots .
$$

Together with (6.13), we have

$$
V \cdot \leq V_{\pi_{n, k}} \text { for any } n, k .
$$

As $k \rightarrow \infty,(6.16)$ and (6.17) imply

$$
V \leq \hat{V}_{n}=\hat{V} .
$$

This completes the proof of Theorem 6.1, since we can see the converse inequality in the same way.

Now we will verify the principle of dynamic programming for $V$ with a tame terminal function $\varphi$.

THEOREM 6.2. $\quad V$ satisfies the principle of dynamic programming, namely

$$
V(t+s, \eta, \varphi)=V(t, \eta, V(s, \cdot, \varphi)) .
$$

Proof. Suppose that $\pi_{n}<\pi_{n+1}$ and $\left\|\pi_{n}\right\| \rightarrow 0$. Appealing to Theorem 6.1, we may assume $t \in \mathscr{P}_{\pi_{n}}, n=1,2, \cdots$, for simplicity. Now the principle of dynamic programming (5.29) yields

$$
V_{n}(t+s, \eta, \varphi)=V_{n}\left(t, \eta, V_{n}(s, \cdot, \varphi)\right)
$$

where $V_{n}=V_{\pi_{n}}$.

On the other hand $V_{n}(, \varphi)$ is decreasing to $V(, \varphi)$ uniformly in any bounded set of $[0, T] \times H$. Thus, for any $\varepsilon>0$ and $r>0$, there is a large $N=N(\varepsilon, r)$, such that, for $n>N$,

$$
\begin{aligned}
& \left|V_{\pi}\left(t, \eta, V_{n}(s, \cdot, \varphi)\right)-V_{\pi}(t, \eta, V(s, \cdot, \varphi))\right| \\
\leq & \sup _{Y, Z}\left|J\left(t, \eta, Y, Z, V_{n}(s, \cdot, \varphi)\right)-J(t, \eta, Y, Z, V(s, \cdot, \varphi))\right|
\end{aligned}
$$




$$
\begin{aligned}
& \leq \sup _{Y, Z} E\left|V_{n}(s, \xi(t, \eta, Y, Z), \varphi)-V(s, \xi(t, \eta, Y, Z), \varphi)\right| \\
& \leq \varepsilon+k_{3}(\varphi) \sup _{Y, Z} E(1+\|\xi(t, \eta, Y, Z)\| ;\|\xi(t, \eta, Y, Z)\| \geq r) \\
& \leq \varepsilon+k_{4}(\varphi)(1+\|\eta\|)\|\eta\| r^{-1}
\end{aligned}
$$

holds. So, as $n \rightarrow \infty, V_{\pi}\left(, V_{n}(s,, \varphi)\right)$ is decreasing to $V_{\pi}(, V(s,, \varphi))$ uniformly in any bounded set of $[0, T] \times H$ and partition $\pi$. This fact together with (6.2), implies

$$
V_{n}\left(t, \eta, V_{n}(s, \cdot \varphi)\right) \rightarrow V(t, \eta, V(s, \cdot, \varphi)) \text { for any } t \text { and } \eta
$$

Now we can conclude the proof by (6.20).

Remark. We introduce the lower value function in the same way as the upper one, putting

$$
W_{\pi}(t, \eta, \Phi)=\sup _{\alpha \in \Gamma_{\pi}} \inf _{Z \in \mathcal{N}} J(t, \eta, \alpha, Z, \Phi)
$$

Under the same condition of Theorem $6.1, W_{\pi_{n}}$ is increasing to $W$, defined by

$$
W(t, \eta, \Phi)=\sup _{\alpha \in \cup \Gamma_{\pi_{n}}} \inf _{Z \in \mathcal{N}} J(t, \eta, \alpha, Z, \Phi) .
$$

For a tame terminal function $\varphi, W(t, \eta, \varphi)$ is independent of a sequence $\left\{\pi_{n}\right\}$ and (6.19) holds.

When $V$ and $W$ coincide, we call it the value function.

\section{Viscosity solution of min-max equation}

We will study the Cauchy problem of min-max equation (7.1) arising in our stochastic differential game.

$$
\left\{\begin{array}{l}
\frac{\partial}{\partial t} u(t, \eta)+F\left(D^{2} u(t, \eta), D u(t, \eta), \eta\right)=0 \text { in }(0, T) \times H \\
u(0)=\varphi, \text { (= tame function) }
\end{array}\right.
$$

where $D=$ Fréchet derivative in $H$ and

$$
=-\min _{z \in \mathscr{Z}} \max _{y \in \mathscr{Y}}\left[\frac{1}{2}(S f(y, z) \eta, f(y, z) \eta)+\langle p,(L(y, z)-c I) \eta\rangle+h(\eta, y, z)\right]
$$




$$
=\max _{z \in \mathscr{Z}} \min _{y \in \mathscr{Y}}\left[\frac{1}{2}(-S f(y, z) \eta, f(y, z) \eta)+\langle p, A(y, z) \eta\rangle-h(\eta, y, z)\right]
$$

where $\langle\rangle=$, duality pairing between $H^{2}$ and $H^{-2}$ under $H=H^{*}$ and $A(y, z)=$ $-L(y, z)+c I$.

Now we will define a viscosity subsolution (resp. supersolution) of (7.1), according to [2. part 4] and [8. part 2]. Since the min-max equation is more general than Bellman equation, our definition is slightly different from theirs. Let us set

$$
\mathscr{D}=\left\{\phi \in C^{12}((0, T) \times H) \cap C([0, T] \times H)\right. \text {; weakly lower }
$$

semi-continuous (WLSC in short) and the following (7.3) (7.5) hold,

(7.3) $\frac{\partial \psi}{\partial t}$ is Lipschitz continuous

(7.4) $D \psi$ is Lipschitz continuous and a continuous mapping from $(0, T) \times H$ into $H^{2}$ with $\sup _{t \leq T}\|D \psi(t, \cdot)\|_{2}<\infty$

(7.5) $\left(D^{2} \phi(t, \zeta) \eta, \eta\right)$ is uniformly continuous in any bounded set of $(0, T)$ $\times H \times H\}$.

Definition 7.1. $u \in \mathrm{UC}_{\mathrm{loc}}([0, T) \times H)$ is called a viscosity subsolution of (7.1) if

(7.6) $u$ is WUSC (weakly upper semi-continuous) on $(0, T) \times H$ and linear growth,

(7.7) for any $\lambda>0$ and $\phi \in \mathscr{D}$, the following inequality holds at each global maximum point $(t, \zeta) \in(0, T) \times H$ of $u(\theta, \eta)-\phi(\theta, \eta)-\lambda\|\eta\|^{2}$,

$$
\frac{\partial \psi}{\partial t}(t, \zeta)+F\left(D^{2} \phi(t, \zeta)+2 \lambda I, D \phi(t, \zeta), \zeta\right) \leq-2 \lambda\left(c-\lambda_{0}\right)\|\zeta\|^{2}
$$

with $\lambda_{0}$ of (2.7), and

$$
u(0)=\varphi
$$

Definition 7.2. $u \in \mathrm{UC}_{\text {loc }}([0, T) \times H)$ is called a supersolution of (7.1), if (7.9) $u$ is WLSC on $(0, T) \times H$ and linear growth,

(7.10) for any $\lambda>0$ and $\phi \in \mathscr{D}$, the following inequality holds at each global minimum point $(t, \zeta) \in(0, T) \times H$ of $u(\theta, \eta)+\phi(\theta, \eta)+\lambda\|\eta\|^{2}$,

$$
-\frac{\partial \psi}{\partial t}(t, \zeta)+F\left(-D^{2} \psi(t, \zeta)-2 \lambda I,-D \phi(t, \zeta), \zeta\right) \geq 2 \lambda\left(c-\lambda_{0}\right)\|\zeta\|^{2}
$$

with $\lambda_{0}$ of (2.7), and 


$$
u(0)=\varphi
$$

$u$ is a viscosity solution, if it is both a viscosity subsolution and a viscosity supersolution. Hereafter, we suppress viscosity, for simplicity, and call viscosity sub- and super-solutions just sub- and super-solutions, respectively.

Now we state the following theorem.

THEOREM 7.1. The upper value function $V(, \varphi)$ is a viscosity solution of min-max equation (7.1).

Proof. Since $V \in \operatorname{LUC}([0, T] \times H) \cap C\left([0, T] \times H_{w}\right), V(\cdot)-\lambda\|\cdot\|^{2}$ is weakly USC and bounded above. Suppose that $\phi \in \mathscr{D}$ and $V(\theta, \eta)-\phi(\theta, \eta)-$ $\lambda\|\eta\|^{2}$ has a global maximum at $(t, \zeta) \in(0, T) \times H$. Now let us assume that

$$
V(\cdot)=V(\cdot, \varphi)=\lim V_{\pi_{n}}(\cdot, \varphi),
$$

recalling Theorem 6.1. Since $V$ satisfies the principle of dynamic programming, we have

$$
\begin{aligned}
& \text { 7.13) } 0=\inf _{\beta \in \cup \Delta_{\pi_{n}}} \sup _{Y \in M} E \int_{0}^{\theta} h(\xi(\tau), Y, \beta) d \tau+V(t-\theta, \xi(\theta))-V(t, \zeta) \\
& \leq \inf _{\beta} \sup _{Y} E \int_{0}^{\theta} h(\xi(\tau), Y, \beta) d \tau+\phi(t-\theta, \xi(\theta))-\phi(t, \zeta)+\lambda\left(\|\xi(\theta)\|^{2}-\|\zeta\|^{2}\right)
\end{aligned}
$$

where $\xi(\tau)=\xi(\tau, \zeta, Y, \beta)$. Thus, Itô's formula yields

$$
\begin{aligned}
0 \leq & \inf _{\beta} \sup _{Y} E\left[\int_{0}^{\theta} h(\xi(\tau), Y, \beta)-\frac{\partial \psi}{\partial t}(t-\tau, \xi(\tau))\right. \\
& -\langle D \phi(t-\tau, \xi(\tau)), A(Y, \beta) \xi(\tau)\rangle \\
& +\frac{1}{2}\left(D^{2} \phi(t-\tau, \xi(\tau))(f(Y, \beta) \xi(\tau)), f(Y, \beta) \xi(\tau)\right) \\
& \left.-2 \lambda\langle\xi(\tau), A(Y, \beta) \xi(\tau)\rangle+\|f(Y, \beta) \xi(\tau)\|^{2} d \tau\right] .
\end{aligned}
$$

Using (7.3) (7.5) and Proposition 3.1, we evaluate each term of (7.14), namely we have $(7.15) \sim(7.22)$ below, putting $m=\sup _{t \leq T}\|D \varphi(t, \cdot)\|_{2}, a=\frac{\partial \phi}{\partial t}(t, \zeta), p=$ $D \phi(t, \zeta), S=D^{2} \phi(t, \zeta)$ and $M\left(\tau, \zeta^{2}\right)=\tau\|\zeta\|^{2}+\int g_{2}(x, \tau)\left\|\Delta_{x} \zeta\right\|^{2} d x$.

$$
E\left|\frac{\partial \Psi}{\partial t}(t-\tau, \xi(\tau))-a\right| \leq k_{1}(|\tau|+M(\tau, \zeta))
$$




$$
\begin{aligned}
& E|\langle D \phi(t-\tau, \xi(\tau)), A(Y, \beta)(\xi(\tau)-\zeta)\rangle| \\
& \leq E \| D \phi\left(t-\tau, \xi(\tau)\left\|_{2}\right\| A(Y, \beta)(\xi(\tau)-\zeta) \|_{-2}\right. \\
& \leq k_{2} m E\|\xi(\tau)-\zeta\| \leq k_{3} M(\tau, \zeta) .
\end{aligned}
$$

Since, for any $\varepsilon>0$, there is $\delta=\delta(\varepsilon, t, \zeta)>0$ such that $\|D \phi(t-\tau, \eta)-p\|_{2}$ $<\varepsilon$, whenever $|\tau|<\delta$ and $\|\eta-\phi\|<\delta$, we get

$$
\begin{aligned}
E \|\left\langle D \phi(t-\tau, \xi(\tau))-p \|_{2}\right. & <\varepsilon+2 m P(\|\xi(\tau)-\zeta\|\rangle \delta) \\
& <\varepsilon+2 m M^{2}(\tau, \zeta) / \delta^{2}
\end{aligned}
$$

Denoting the Lipschitz constant of $D \phi$ by $r$, we see

$$
\left|\left(D^{2} \phi(\theta, \xi) \eta, \eta\right)\right| \leq r\|\eta\|^{2} .
$$

Since, for any $\varepsilon>0$ and $R>0$, there is $\delta_{0}=\delta_{0}(\varepsilon, R)>0$ such that

$$
\left|\left(D^{2} \phi(\theta, \xi) \eta, \eta\right)-\left(D^{2} \phi(\tilde{\theta}, \tilde{\xi}) \tilde{\eta}, \tilde{\eta}\right)\right|<\varepsilon
$$

whenever $|\theta-\tilde{\theta}|<\delta_{0},\|\xi-\tilde{\xi}\|<\delta_{0},\|\eta-\tilde{\eta}\|<\delta_{0}$, and $\|\xi\|,\|\tilde{\xi}\|,\|\eta\|,\|\tilde{\eta}\| \leq R$, we get

$$
\begin{aligned}
& E \mid\left(D^{2} \phi(t-\tau, \xi(\tau))(f(Y, \beta) \zeta), f(Y, \beta) \zeta\right)-(S(f(Y, \beta) \zeta, f(Y, \beta) \zeta) \mid \\
& <\varepsilon+2 r|f|_{\infty}^{2}\|\zeta\|^{2}\left(P(\|\xi(\tau)\|>R)+P\left(\|\xi(\tau)-\zeta\|>\delta_{0}\right)\right) \\
& <\varepsilon+2 r|f|_{\infty}^{2}\|\zeta\|^{2}\left(\left(E\|\xi(\tau)\|^{2} / R^{2}\right)+\left(M^{2}(\tau, \zeta) / \delta_{0}^{2}\right)\right)
\end{aligned}
$$

for a large $R>|f|_{\infty}\|\zeta\|$.

$$
\begin{aligned}
& E \mid\left(D^{2} \phi(t-\tau, \xi(\tau))(f(Y, \beta) \xi(\tau)), f(Y, \beta) \xi(\tau)\right) \\
& -\left(D^{2} \psi(t-\tau, \xi(\tau))(f(Y, \beta) \zeta), f(Y, \beta) \zeta\right) \mid \\
& <r|f|_{\infty}^{2}\left(E\|\xi(\tau)-\zeta\|^{2}+2\|\zeta\| E\|\xi(\tau)-\zeta\|\right) \\
& <r|f|_{\infty}^{2}\left(M^{2}(\tau, \zeta)+2\|\zeta\| M(\tau, \zeta)\right) \text {. } \\
& (7.21) \quad-2 \lambda E\langle\xi(\tau), A(Y, \beta) \xi(\tau)\rangle \\
& =-2 \lambda E\left[c\|\xi(\tau)\|^{2}-\langle\xi(\tau), L(Y, \beta) \xi(\tau)\rangle\right] \\
& \leq 2 \lambda\left(\lambda_{0}-c\right) E\|\xi(\tau)\|^{2}=2 \lambda\left(\lambda_{0}-c\right)\left[\|\zeta\|^{2}+E\left(\|\xi(\tau)\|^{2}-\|\zeta\|^{2}\right)\right] \\
& \leq 2 \lambda\left(\lambda_{0}-c\right)\|\zeta\|^{2}+k_{4}\|\zeta\| M(\tau, \zeta) \text {. }
\end{aligned}
$$

$$
E \mid \| f(Y, \beta) \zeta) \xi(\tau)\left\|^{2}-\right\| f(Y, \beta) \zeta\left\|^{2} \mid \leq k_{5}\right\| \zeta \| M(\tau, \zeta) .
$$


Inserting above (7.15) (7.22) into (7.14), we obtain

$$
\begin{aligned}
0 & \leq \inf _{\beta} \sup _{Y} E\left[\int_{0}^{\theta} h(\zeta, Y, \beta)-a-\langle p, A(Y, \beta) \zeta\rangle\right. \\
& \left.+\frac{1}{2}((S+2 \lambda I) f(Y, \beta) \zeta, f(Y, \beta) \zeta) d \tau\right] \\
& +2 \lambda\left(\lambda_{0}-c\right)\|\zeta\|^{2} \theta+o(\theta) .
\end{aligned}
$$

Next we calculate the main term of (7.23), putting

$$
\begin{aligned}
K(y, z)=h(\zeta, y, z)-a-\langle p, & A(y, z) \zeta\rangle \\
& +\frac{1}{2}((S+2 \lambda I) f(y, z) \zeta, f(y, z) \zeta) .
\end{aligned}
$$

Since $\zeta$ is fixed, $K(y, z)$ is constant in $H$. So, it can be regarded as a tame function. Let us consider a differential game, using $K$ instead of $h$. Putting

$$
\tilde{V}_{\pi}(\theta, \eta, \varphi)=\inf _{\beta \in \Delta_{\pi}} \sup _{Y \in \mathcal{M}} E \int_{0}^{\theta} K(Y(s), \beta(Y)(s)) d s+\varphi(\xi(\theta, \eta, Y, \beta))
$$

and $\mathscr{P}_{\pi}=\left\{t_{p}, p=0, \cdots, N\right\}$, the principle of dynamic programming asserts

$$
\tilde{V}_{\pi}(\theta, \eta, \varphi)=\tilde{V}_{\pi}\left(t_{p}, \eta, \tilde{V}_{\pi\left(t_{p}\right)}\left(\theta-t_{p}, \cdot, \varphi\right)\right) .
$$

Setting $\varphi=0$, we have, for $\theta \in\left[t_{p}, t_{p+1}\right)$ and $\tilde{\zeta} \in H$

$$
\tilde{V}_{\pi\left(t_{p}\right)}\left(\theta-t_{p}, \tilde{\zeta}, 0\right)=\min _{z \in \mathscr{Z}} \sup _{Y \in \mathcal{M}} E \int_{0}^{\theta-t_{p}} K(Y(s), z) d s .
$$

Noting

$$
\begin{gathered}
\sup _{Y \in \mathcal{M}} E \int_{0}^{t} K(Y(s), z) d s \leq \int_{0}^{t} \max _{Y \in \mathscr{Y}} K(Y, z) d s=\max _{y \in \mathscr{Y}} K(y, z) t \\
=\max _{y \in \mathscr{Y}} E \int_{0}^{t} K(y, z) d s \leq \sup _{Y \in \mathscr{M}} E \int_{0}^{t} K(Y(s), z) d s,
\end{gathered}
$$

we get

$$
\tilde{V}_{\pi\left(t_{p}\right)}\left(\theta-t_{p}, \hat{\zeta}, 0\right)=\min _{z \in \mathscr{Z}} \max _{Y \in \mathscr{Y}} K(y, z)\left(\theta-t_{p}\right)=\mu\left(\theta-t_{p}\right)
$$

where $\mu=\min _{z \in \mathscr{Z}} \max _{Y \in \mathscr{Y}} K(y, z)$. Again it follows from (5.39) that

$$
\tilde{V}_{\pi}\left(t_{p}, \eta, \mu\left(\theta-t_{p}\right)\right)=\tilde{V}_{\pi}\left(t_{p-1}, \eta, \hat{V}_{\pi\left(t_{p-1}\right)}\left(t_{p}-t_{p-1}, \cdot, \mu\left(\theta-t_{p}\right)\right)\right. \text {, }
$$


and

$$
\tilde{V}_{\pi}\left(t_{p-1}\right)\left(t, \tilde{\zeta}, \mu\left(\theta-t_{p}\right)\right)=\mu t+\mu\left(\theta-t_{p}\right) .
$$

Thus, we obtain

$$
\tilde{V}_{\pi}(\theta, \eta, 0)=\mu \theta
$$

Therefore

$$
\inf _{\beta} \sup _{Y} E \int_{0}^{\theta} K(Y(s), \beta(Y)(s)) d s=\lim _{n \rightarrow \infty} \tilde{V}_{\pi_{n}}(\theta, \eta, 0)=\mu \theta,
$$

holds. Now, (7.23) concludes

$$
-2 \lambda\left(\lambda_{0}-c\right)\|\zeta\|^{2} \leq \mu \theta .
$$

Recalling the definition of $\mu,(7.32)$ asserts that $V$ is a subsolution.

Since we can prove that $V$ is a supersolution in the similar way, the proof is completed.

In the finite dimensional case (see [3], [5]), a subsolution (resp. supersolution) can be defined by super differential (resp. subdifferential) instead of test functions. But, in our case, this equivalence is open. Here we remark on super differential $J^{+} u$ (resp. subdifferential $J^{-} u$ ) for $u \in \mathrm{UC}_{\mathrm{loc}}((0, T) \times H) \cdot J^{+} u$ is defined by (7.33)

$$
\begin{gathered}
J^{+} u(t, \zeta)=\left\{(a, p, S) \in R^{1} \times H^{2} \times \mathscr{L}\right. \\
\left.u(t+\theta, \zeta+\eta) \leq u(t, \zeta)+a \theta+(p, \eta)+\frac{1}{2}(S \eta, \eta)+o\left(|\theta|+\|\eta\|^{2}\right)\right\} \\
\qquad \text { for }(t, \zeta) \in(0, T) \times H,
\end{gathered}
$$

where $\mathscr{L}=$ space of all continuous linear operators from $H$ to $H^{*}(=H)\left(J^{-}(u)\right.$ is defined by (7.33) with the opposite inequality).

Remark. Let $u \in \mathrm{UC}_{\mathrm{loc}}([0, T) \times H)$ be weakly USC and bounded above, say $M=\sup _{\theta, \eta} u(\theta, \eta)$. Suppose

$$
\sup _{\theta \in[0, T]} u(\theta, \eta) \rightarrow-\infty, \text { as }\|\eta\| \rightarrow \infty .
$$

Let us fix $(t, \zeta) \in(0, T) \times H$ and $\delta>0$ arbitrarily. Then, for any $\alpha>$ $(M-u(t, \zeta)+1) / \delta$,

$$
u(\theta, \eta)-\alpha\left(\|\eta-\zeta\|_{-1}^{2}+|\theta-t|^{2}\right)<u(t, \zeta)-1
$$


holds, whenever $\|\eta-\zeta\|_{-1}^{2}+|\theta-t|^{2}>\delta$. On the other hand, it follows from (7.34) that there is a positive number $m$, such that

$$
u(\theta, \eta)<u(t, \zeta)-1, \quad \text { if }\|\eta\|>m .
$$

But, \|\|$_{-1}$ is WLSC, because $\|\eta\|_{-1}=\sup \left\{(\eta, e) ; e \in H_{1}\right.$ and $\left.\|e\|=1\right\}$. Hence $u-\alpha\left(\|\cdot-\zeta\|_{-1}^{2}+|\cdot-t|^{2}\right)$ has a global maximum point $(\tilde{\theta}, \tilde{\zeta})$, such that

$$
\|\tilde{\zeta}\| \leq m \text { and }\|\tilde{\zeta}-\zeta\|_{-1}^{2}+|\tilde{\theta}-t|^{2}<\delta .
$$

Therefore, setting $B=(I-\Delta)^{-1}$, where $\Delta=$ Laplacian operator,

$u(\tilde{\theta}+\theta, \tilde{\zeta}+\eta) \leq u(\tilde{\theta}, \tilde{\zeta})+2 \alpha(\tilde{\theta}-t) \theta+2 \alpha(B(\tilde{\zeta}-\zeta), \eta)+\alpha(B \eta, \eta)+\alpha \theta^{2}$ holds. Noting $B(\tilde{\zeta}-\zeta) \in H^{2}$ and $B \in \mathscr{L}$ we get

$$
(2 \alpha(\tilde{\theta}-t), 2 \alpha B(\tilde{\zeta}-\zeta), \alpha B) \in J^{+} u(\tilde{\theta}, \tilde{\zeta}) .
$$

Since $t, \zeta$ and $\delta$ are arbitrary, (7.37) and (7.38) yield that $\{(\theta, \eta) \in(0, T) \times H$; $\left.J^{+} u(\theta, \eta) \neq \phi\right\}$ is dense in $R^{1} \times H^{-1}$-topology.

\section{Example}

In this section, we will deal with a simple example, where the upper value function is a unique viscosity solution in a class of finite dimensional functions on $H$.

Setting $\partial_{i}=\frac{\partial}{\partial x_{i}}$, let us consider $\operatorname{SPDE}(8.1)$ below,

$$
\begin{aligned}
d \xi(x, t)= & \sum_{i=1}^{n} \sigma^{i}(Y(t), Z(t))\left(\partial_{i}^{2}-\left(x_{i}^{2} / 4\right)\right) \xi(x, t) d t \\
& +f(Y(t), Z(t)) \xi(x, t) d W(t),
\end{aligned}
$$

with initial condition

$$
\xi(x, 0)=\eta(x) .
$$

Although SPDE (8.1) does not satisfy the condition; $c=$ constant, we can easily see the same results, using an Hermite base of $H$. Let us assume (a1) (a3) below. (a1) $\sigma^{i}(y, z)$ and $f(y, z)$ are Lipschitz continuous in $\mathscr{Y} \times \mathscr{Z}$,

(a2) there is a positive number $\lambda^{*}$ such that

$$
\sigma^{\mathrm{i}}(y, z) \geq \lambda^{*} \text { for any } y \text { and } z
$$

(a3) $h(\cdot, y, z)$ and an initial function $\varphi$ are tame functions of Hermite base $\boldsymbol{e}_{\underline{k}}$ 
and bounded.

Here the Hermite base is defined as follows. For a multi-index $\underline{k}=\left(k_{1}, \cdots, k_{n}\right)$, $k_{i} \geq 0$.

$$
\boldsymbol{e}_{\underline{k}}(\underline{x})=\prod_{i=1}^{n} \boldsymbol{e}_{k_{i}}\left(x_{i}\right) \quad \text { for } \underline{x}=\left(x_{1}, \cdots, x_{n}\right) \in R^{n},
$$

where $\boldsymbol{e}_{0}(x)=(2 \pi)^{-1 / 4} \exp \left(-\frac{x^{2}}{4}\right)$ for $x \in R^{1}$, and

$$
\boldsymbol{e}_{m}(x)=(-1)^{m}(\sqrt{2 \pi} m !)^{-1 / 2}\left[\exp \left(\frac{x^{2}}{4}\right)\right]\left(\frac{d}{d x}\right)^{m} \exp \left(\frac{-x^{2}}{2}\right) \text { for } x \in R^{1}, m=1,2, \cdots
$$

Appealing to the following fact,

$$
\frac{d}{d x} \boldsymbol{e}_{m}(x)=\frac{1}{2}\left(\sqrt{m+1} \boldsymbol{e}_{m+1}(x)-\sqrt{m} \boldsymbol{e}_{m-1}(x)\right),
$$

we have, for $\eta=\sum c_{\underline{k}} \boldsymbol{e}_{\underline{k}} \in H$,

$$
\partial_{i} \eta=\frac{1}{2}\left(\sum c_{\underline{k}} \sqrt{k_{i}+1} \boldsymbol{e}_{\underline{k}+I_{i}}-\sum c_{\underline{k}} \boldsymbol{e}_{\underline{k}-I_{i}}\right)
$$

where $\underline{k} \pm I_{i}=\left(k_{1}, \cdots, k_{i} \pm 1, \cdots, k_{n}\right)$. Therefore $\partial_{i} \eta \in H$, if and only if $\sum c_{\underline{k}}^{2} k_{i}<\infty$. Repeating this argument, we see

Proposition 8.1.

If $\sum c_{\underline{k}}^{2}|\underline{k}|^{p}<\infty$, then $\eta \in H^{p}, p=0,1,2, \cdots$.

Moreover, $\boldsymbol{e}_{m}$ satisfies the following equation

$$
\boldsymbol{e}_{m}^{\prime \prime}-\left(x^{2} / 4\right) \boldsymbol{e}_{m}=-\left(m+\frac{1}{2}\right) \boldsymbol{e}_{m}, \quad m=0,1,2, \cdots
$$

Hence, employing the formal expantion of $\xi(t)$, say

$$
\xi(t)=\sum X_{\underline{k}}(t) \boldsymbol{e}_{\underline{k}}
$$

we have SDE

$$
\left\{\begin{array}{l}
d X_{\underline{k}}(t)=-\left(\sum_{i=1}^{n} \sigma^{i}(Y(t), Z(t))\left(k_{i}+\frac{1}{2}\right)\right) d t+f(Y(t), Z(t)) X_{\underline{k}}(t) d W(t) \\
X_{\underline{k}}(0)=\left(\eta, \boldsymbol{e}_{\underline{k}}\right) .
\end{array}\right.
$$

(8.6) has a unique solution with continuous path and 


$$
E\left|X_{\underline{k}}(t)\right|^{2} \leq\left|X_{\underline{k}}(0)\right|^{2} \exp \left(-2 \lambda^{*}\left(|k|+\frac{n}{2}+|f|_{\infty}^{2}\right) t\right)
$$

holds, where $|k|=\sum k_{i}$ and $|f|_{\infty}=\sup _{y, z}|f(y, z)|$.

Now for $\xi$ of (8.5), we can easily see that $\xi \in L^{2}\left(\Omega \times(0, T) ; H^{2}\right) \cap L^{2}(\Omega$; $C([0, T] ; H))$ and $\xi$ provides a unique solution of $(8.1)$. Moreover, the evaluation (2.3) (2.5) and Proposition 3.1 hold. Therefore the upper value function $V(\cdot, \varphi)$ is a viscosity solution of the min-max equation (8.8) below.

$$
\left\{\begin{array}{l}
\frac{\partial u}{\partial t}(t, \eta)-\min _{z \in \mathscr{Z}} \max _{y \in \mathscr{Y}}\left[\frac{1}{2}|f(y, z)|^{2}\left(D^{2} u(t, \eta) \eta, \eta\right)\right. \\
\quad+\langle D u(t, \eta), L(y, z) \eta\rangle+h(\eta, y, z)]=0 \\
u(0, \eta)=\varphi(\eta),
\end{array}\right.
$$

where

$$
L(y, z)=\sum_{i=1}^{n} \sigma^{i}(y, z)\left(\partial_{i}^{2}-\left(x_{i}^{2} / 4\right)\right) .
$$

By (a3), $h$ and $\varphi$ can be written by

$$
h(\eta, y, z)=\hat{h}\left(\left(\eta, \boldsymbol{e}_{1}\right), \cdots,\left(\eta, \boldsymbol{e}_{N}\right), y, z\right)
$$

and

$$
\varphi(\eta)=\hat{\varphi}\left(\left(\eta, \boldsymbol{e}_{1}\right), \cdots,\left(\eta, \boldsymbol{e}_{N}\right)\right)
$$

where $\boldsymbol{e}_{j}=\boldsymbol{e}_{\underline{k}}$ for $\underline{k}=\underline{k}^{j}=\left(k_{1}^{j}, \cdots, k_{n}^{j}\right)$. Let us set $P=$ orthogonal projection onto the linear space generated by $\left(\boldsymbol{e}_{1}, \cdots, \boldsymbol{e}_{N}\right)$. Then, (8.6) derives that $V(t, \eta, \varphi)$ depends only on $\left(\left(\eta, \boldsymbol{e}_{1}\right), \cdots,\left(\eta, \boldsymbol{e}_{N}\right)\right)$, namely

$$
V(t, \eta, \varphi)=V(t, P \eta, \varphi)
$$

Define $\boldsymbol{v}$ by

$$
\boldsymbol{v}\left(t, b_{1}, \cdots, b_{N}\right)=V\left(t, b_{1} \boldsymbol{e}_{1}+\cdots+b_{N} \boldsymbol{e}_{N} ; \varphi\right) .
$$

Again (8.6) implies that $\boldsymbol{v}$ is a unique viscosity solution of the following finite dimensional min-max equation (8.12), and bounded by (a3),

$$
\left\{\begin{array}{l}
\frac{\partial \boldsymbol{v}}{\partial t}(t, b)-\min _{z} \max _{y}\left[\frac{1}{2} f^{2}(y, z) \sum_{i, j=1}^{N} \frac{\partial^{2} v}{\partial b_{i} \partial b_{j}}(t, b) b_{i} b_{j}\right. \\
\left.\quad+\sum_{j=1}^{N} \sum_{i=1}^{n} \sigma^{i}(y, z)\left(k_{i}^{j}+\frac{1}{2}\right) b_{j} \frac{\partial v}{\partial b_{j}}(t, b)+\hat{h}(b, y, z)\right]=0 \\
v(0, b)=\hat{\varphi}(b)
\end{array}\right.
$$

From (8.10), we see $V(t, \eta, \varphi)=\boldsymbol{v}\left(t,\left(\eta, \boldsymbol{e}_{1}\right), \cdots,\left(\eta, \boldsymbol{e}_{N}\right)\right)$ 
Theorem 8.1. Suppose that $W \in \mathrm{BUC}([0, T] \times H)$ is a viscosity solution of (8.8) and $W(t, \eta)=W(t, P \eta)$. Then $W=V$.

Proof. Suppose that $\hat{\phi} \in C^{12}\left((0, T) \times R^{N}\right)$ has the two properties below, (i ) $\frac{\partial \hat{\psi}}{\partial t}$ is Lipschitz continuous

and

(ii) $\frac{\partial \hat{\psi}}{\partial x_{1}}$ and $\frac{\partial^{2} \hat{\psi}}{\partial x_{i} \partial x_{j}}$ are bounded.

Put $\phi(t, \eta)=\hat{\phi}\left(t,\left(\eta, \boldsymbol{e}_{1}\right), \cdots,\left(\eta, \boldsymbol{e}_{N}\right)\right)$. Then $\phi \in \mathscr{D}$ by (i) and (ii).

The function $\hat{w}$, defined by

$$
\hat{w}\left(t, b_{1}, \cdots, b_{N}\right)=W\left(t, b_{1} \boldsymbol{e}_{1}+\cdots+b_{N} \boldsymbol{e}_{N}\right)
$$

is continuous, since $W$ is weakly continuous, and

$$
W(t, \eta)=\hat{w}\left(t,\left(\eta, e_{1}\right), \cdots,\left(\eta, e_{N}\right)\right)
$$

holds.

Soppose that $\hat{w}-\hat{\phi}$ has a unique maximum at $(\hat{t}, \hat{b}) \in(0, T) \times R^{N}$, and $W(t, \eta)-\phi(t, \eta)-\lambda\|\eta\|^{2}$ has a global maximum at $\left(t_{\lambda}, \eta_{\lambda}\right)$. Appealing to $\|P \eta\| \leq\|\eta\|$, we have

$$
W(t, \eta)-\phi(t, \eta)-\lambda\|\eta\|^{2} \leq W\left(t_{\lambda}, P \eta_{\lambda}\right)-\phi\left(t_{\lambda}, P \eta_{\lambda}\right)-\lambda\left\|P \eta_{\lambda}\right\|^{2} .
$$

So, we may assume $P \eta_{\lambda}=\eta_{\lambda}$. Therefore, as $\lambda \rightarrow 0, t_{\lambda}$ and $\left(\eta_{\lambda}, \boldsymbol{e}_{j}\right)$ tend to $\hat{t}$ and $\hat{b}_{j}$ respectively and $t_{\lambda}>0$ for small $\lambda$. Fixing $\lambda$ arbitrarily and putting $t_{\lambda}=s$, $\left(\eta_{\lambda}, \boldsymbol{e}_{j}\right)=\beta_{j}$ and $\beta=\left(\beta_{1}, \cdots, \beta_{N}\right)$, we can easily see

$$
\begin{aligned}
& \frac{\partial \hat{\psi}}{\partial s}(s, \beta)-\min _{z} \max _{y}\left[\sum_{k, j=1}^{N} \frac{1}{2} f^{2}(y, z) \frac{\partial^{2} \hat{\psi}(s, \beta)}{\partial \beta_{k} \partial \beta_{j}} \beta_{k} \beta_{j}+\lambda|\beta|^{2}\right. \\
& \left.\quad+\sum_{j=1}^{N} \sum_{i=1}^{n} \sigma^{i}(y, z)\left(k_{i}^{j}+\frac{1}{2}\right) \beta_{j} \frac{\partial \hat{\psi}(s, \beta)}{\partial \beta_{j}}+\hat{h}(\beta, y, z)\right] \\
& \leq 2 \lambda \lambda_{0}|\beta|^{2} .
\end{aligned}
$$

Tending $\lambda$ to 0 , (8.15) imllies that $\hat{w}$ is a subsolution of (8.12).

By the same argument, we can show that $\hat{w}$ is a super solution. Hence we get $\hat{w}=\boldsymbol{v}$. Recalling (8.13), we conclude the proof. 


\section{REFERENCES}

[1] D.G. Aronson, Non-negative solution of linear parabolic equation, Ann. della Scuola Norm. Sup. Pisa, 22 (1968), 607-694.

[2] M.G. Crandall and P.L. Lions, Hamilton-Jacobi equations in infinite dimensions, Part 4, J. Funct. Anal., 90 (1990), 273-283, Part 5, 97 (1991), 417-465.

[ 3 ] M.G. Crandall, H. Ishii and P.L. Lions, A user's guide to viscosity solutions, Bull. Amer. Math. Soc., N. S., 27 (1992), 1-67.

[4] W.H. Fleming and P.E. Souganidis, On the existence of value function of two-player, zero-sum stochastic differential games, Indiana Univ. Math. J., 38, (1989), 293-314.

[5] W.H. Fleming and H.M. Soner, Controlled Markov Processes and Viscosity Solutions, Springer Verlag, 1992.

[6] H. Ishii, Viscosity solution of nonlinear second-order partial differential equation in Hilbert spaces, Preprint.

[ 7 ] N.V. Krylov and B.L. Rozovskii. On the Cauchy problem for linear stochatic partial differential equations, Math. USSR Izv., 11 (1977), 1267-1284.

[8] P.L. Lions, Viscosity solutions and optimal stochastic control in infinite dimensions, Part 1, Acta Math., 161 (1988), 243-278, Part 2 L. N. M., 1390 (1988), 147-170, Part 3, J. Funct. Anal., 86 (1989), 1-18.

[9] M. Nisio, Optimal control for stochastic differential equations and viscosity solutions of Bellman equations, Nagoya Math. J., 123 (1991), 13-37.

[10] E. Pardoux, Stochastic partial differential equations and filtering of diffusion processes, Stochastics, 3 (1979), 127-167.

W.H. Fleming

Division of Applied Mathematics

Brown University

Providence R. I,. 02912, USA

M. Nisio

Department of Mathematics

Kobe University

Rokko, Kobe 657, Japan

Current address:

Department of Mathematics

Osaka Electro-Communication University

Hatsu-cho, Neyagawa 572, Japan 